\title{
Capacity pooling games in crowdsourcing services
}

\author{
Zhanwen Shi ${ }^{1,2} \cdot \mathrm{Erbao}^{\mathrm{CaO}}{ }^{2,3} \cdot \mathrm{Kai} \mathrm{Nie}^{2,3}$
}

Accepted: 13 July 2021

(c) The Author(s), under exclusive licence to Springer Science+Business Media, LLC, part of Springer Nature 2021

\begin{abstract}
In crowdsourcing services, employers often post some complex (or difficult) tasks that individual workers cannot complete independently. In this paper, we investigate that a group of independent workers willingly form a workers coalition by pooling their capacities together to jointly complete a crowdsourcing task, with the goal of being to obtain a reward from an employer. The capacity pooling games in the crowdsourcing service setting are formulated as optimization problems. Using the duality theory of a linear program, we not only establish that the core of the capacity pooling game is nonempty but also provide a simple way to compute a fair profit allocation policy in the bidding mode, employment mode and contrast mode of crowdsurcing services, respectively. Then, we further analyze the capacity pooling games with concave investment cost and convex quality reward structures, which exhibit the economies of scale and quality incentives. More interestingly, we give a constructive proof to the nonemptiness of the core of the resulting capacity pooling game with nonlinear structures.
\end{abstract}

Keywords Crowdsourcing $\cdot$ Cooperative game $\cdot$ Capacity pooling $\cdot$ Allocation policy $\cdot$ Duality theory

\section{Introduction}

Product and technological innovation has always been an important issue that enterprises focus on. With the rapid development of information level, enterprises can strengthen the direct communication with customers. This interaction has become an interesting source of new product creativity and technological advance. Taking

Kai Nie

niekai@hnu.edu.cn

1 School of Business Administration, Zhongnan University of Economics and Law, Wuhan 430073, China

2 College of Economic and Trade, Hunan University, Changsha 410079, China

3 Key Laboratory of Logistics Information and Simulation Technology, Changsha 410079, China 
advantage of this opportunity, enterprises are now creating online creative markets where consumers can launch new product and technological ideas that could potentially generate revenue for consumers [15]. This new approach is named crowdsourcing by Howe [12], which defines crowd as the new pool of cheap labor from the network. Crowdsourcing accelerates the idea discovery process by expanding the scale and diversity of collective wisdom devoted to problem solving, and offers customers co-creation experience that helps enterprises better reap competitive advantages [5]. Compared with traditional innovation sourcing approaches [14], a promising crowdsourcing service brings the employers (answer-seekers) a number of advantages. For example, employers have access to a crowd of workers (answer-solvers) who may be able to access a wider array of talents, and generate better answers than the ones presented in one organization. Meanwhile, employers tend to only pay for some selected answers, so the cost of seeking answers would be greatly reduced [17]. In addition, the crowdsourcing service also brings a great deal of benefits to workers. The contributors of the answers will be compensated monetarily with reward [1]. Thanks to the Internet, crowdsourcing platforms (e.g., Zhubajie, TaskCN, Kaggle, TopCoder, Crowdspring, DesignCrowd, InnoCentive) have emerged and have been widely applied in various fields $[6,13,18,21]$.

Some popular crowdsourcing platforms (e.g., TaskCN, Zhubajie, DesignCrowd, Threadless, InnoCentive) mainly comprise of three modes of crowdsourcing service, i.e., the bidding mode, the employment mode and the contrast mode. Generally speaking, employers post some unsolved tasks and reward budgets on the crowdsourcing platforms. Workers who have registered on the crowdsourcing platforms choose some their interested tasks to undertake, aiming to achieve rewards from employers by completing and submitting answers of these tasks into the crowdsourcing platforms, which tends to incur some capacity investment costs. Then, employers evaluate those submitted answers and pay rewards for selected answers. Finally, after the whole crowdsourcing transaction is completed, if a certain employer find that the realized quality of the submitted answer becomes inconsistent with the one previously submitted in a time period, the employer could initiate a refund on the crowdsourcing platforms and the named worker would have to refund partial (or all) reward. For instance, an employer finds that his website system developed by a worker often has some problems after a period of use, which deviates from the quality of original submission. At this time, the employer can claim a refund to cover the loss of quality through the crowdsourcing platforms.

It is worth noting that employers often post some complex or difficult tasks (e.g., software development, house decoration, website construction, industrial design) on the crowdsourcing platforms in practice, and individual workers have not enough capacity to complete these tasks independently. In this case, independent workers may choose to willingly form a workers coalition, sometimes also referred to as a team, organization or firm, to jointly work on these tasks in a collaborative way. In other words, this class of tasks would be divided into small pieces that can be accomplished independently by each player of a workers coalition. For example, Netflix released a crowdsourcing task in 2006, which asked the global internet users to develop a recommendation algorithm that is more accurate than Netflix's own algorithm. The winner would obtain a grand prize of $\$ 1,000,000$ from Netflix. 
Initially, no independent workers could achieve this goal required by Netflix; then, different workers began to collaborate to jointly accomplish this difficult task. In the end, the workers coalition, called as Bellkor's Pragmatic Chaos, consisting of seven people completed this task; the workers coalition consisted of statistical experts, machine learning specialists and computer engineers [41]. Google map is a citizen science-oriented crowdsourcing task. A sense of collaboration plays a leading role in this task and a vast number of workers collaborate to tag and update maps [44].

According to the existing literature (e.g., [1, 5, 6, 17, 19, 37, 43]), we find that these literature mainly considered how a typical individual worker could best undertake a crowdsourcing task. Nevertheless, there are few studies looking at collaboration strategies and the capacity pooling effect for workers to accomplish a complex or difficult task using the cooperative game theory in crowdsourcing services. To the best of our knowledge, there are also a limited number of studies on cooperative behaviors among workers (e.g., [13, 22]). However, these studies mainly focus on how an employer should optimally design a crowdsourcing contest for such cooperative behaviors, and neglect the design of fair profit allocation policies for a workers coalition. As a result, two central questions facing a workers coalition are naturally proposed in different modes of crowdsourcing services. First, how do a group of independent workers form a willing workers coalition by pooling their capacities together to jointly complete a crowdsourcing task, with the goal being to obtain a reward? Second, when a workers coalition receives a reward from an employer, how do fairly allocate the profit among players of the workers coalition to motivate independent workers to collaborate? These two questions constitute the key research of this paper.

To answer these questions, we first construct a game-theoretical model to capture the cooperative behavior of a workers coalition when posted a crowdsourcing task. Our model distinguishes three different modes of crowdsourcing services when faced with different types of crowdsourcing tasks. Then, one important issue for a workers coalition is to keep different members motivated to collaborate. The willingness to collaborate often depends on the existence of mechanisms that allocate the profit in such a way that it is considered fair by all members. The notion of fairness in this paper can be captured by the concept of core in the cooperative game theory [31, 34]. Namely, allocating profit according to the cooperative game theory may create incentives for independent workers to form a coalition. Specifically, the cooperative game theory has become one of the systematic tools in analyzing fair allocation mechanisms and has found numerous applications. For example, Chen and Zhang [8] uses the cooperative game theory to analyze the cost allocation issue in an inventory centralization game, Shi and Cao [35] explores a profit allocation policy for the agricultural producers' cooperative game in contract farming using the cooperative game theory. Thus, we model the profit allocation issue for a workers coalition in crowdsourcing services as a cooperative game, referred to as the capacity pooling games in this paper. Finally, we take advantage of a duality approach to investigate whether there exists a profit allocation policy in the core of the capacity pooling game.

The main results of this paper are summarized as follows. First, in the bidding mode of crowdsourcing services, we formulate the capacity pooling game with a 
refund and no refund as a linear programming problem, respectively, and provide a simple way to compute a profit allocation policy in the core of the capacity pooling game using the duality theory. When the workers coalition faces the economies of scale and quality incentives, i.e., the concave investment cost and the convex quality reward, we also demonstrate that the core of the corresponding capacity pooling game is nonempty. Then, we illustrate the duality theory to the capacity pooling games in the employment mode and the contrast mode of crowdsourcing services, respectively. Interestingly, the constructive proofs to the nonemptiness of core of the capacity pooling games in the employment mode and contrast mode are also given whether there is a refund or not.

The remainder of this paper is organized as follows. In Sect. 2, we briefly review some related literature. In Sect. 3, we introduce some basic concepts of the cooperative game theory and formulate the capacity pooling problems in crowdsourcing services. The capacity pooling game in the bidding mode is analyzed in Sect. 4. In Sect. 5, we focus on the capacity pooling games in the employment mode and contrast mode, respectively. We illustrate our proposed results using numerical analyses and conduct sensitivity analyses in Sect. 6. Section 7 concludes this paper and provides some directions for future research.

\section{Literature review}

It is widely known that crowdsourcing has a long history. Well-known early examples of crowdsourcing include the Longitude Prize, established in 1714, and the creation of the Oxford English dictionary, published in 1884 [42]. More generally, the story of those in power (kings, sultans, emperors) offering prizes to anyone who can come up with the best solution to a particular problem appears in historical records and folklore, dating back hundreds and thousands of years [1]. Although the concept of crowdsourcing has existed for many years, the term "crowdsourcing" was first introduced by wired reporter Jeff Howe in 2006 and defined as "the act of a company or institution taking a function once performed by employees and outsourcing it to an undefined network of people in the form of an open call. This can take the form of peer-production, but is also often undertaken by sole individuals" [12]. The ubiquity and low cost of network connection, as well as the emergence of mobile computing, so that employers and platforms can quickly identify and connect agents and employers to complete tasks. So far, crowdsourcing has been used to solve problems, generate ideas and production models. The purpose is to make use of the heterogeneity of the population and its scattered knowledge to better propose answers to complete tasks [10, 24, 40].

In this paper, a group of independent workers willingly form a coalition to jointly undertake a complex or difficult task posted on a crowdsourcing platform by an employer. We mainly focus on collaboration strategies for a workers coalition, with the goal of obtaining a reward from an employer. What's more, we design different profit allocation policies for a workers coalition using the duality theory when there exist a refund and no refund, respectively. Thus, there are three strands of literature that are closely related to our paper. The first group examines the theoretical 
research on crowdsourcing services, which is mainly concentrated on the optimal design of the reward structure and the asymptotic structure of the crowdsourcing service. The second group explores the empirical studies that largely focus on the behavior of workers. The third group comprises studies that could be in a different context from our paper but share methodological similarities.

The first group of literature is related to the theoretical research on the optimal strategies. Considerable research has been devoted to exploring questions connected with crowdsourcing services, see Archak and Sundararajan [3], DiPalantino and Vojnovic [9], Ghosh and McAfee [11], Karger et al. [19], Nevo and Kotlarsky [24], and Pandey et al. [27] for reviews on crowdsourcing. In the earlier works, Terwiesch and $\mathrm{Xu}$ [39] established that the workers facing a performance-contingent reward would like to exert more effort than those facing a fixed-price reward in general. At the same time, they also found that employers tended to benefit greatly from a large number of workers. Archak and Sundararajan [3] paid attention to the strategic behaviors of both risk-neutral and risk-averse workers in crowdsourcing services and found that employers should allocate the whole budget to the top reward when workers were risk-neutral. On the other hand, they also showed that employers might provide more rewards than the number of submissions in the optimal case when workers were risk-averse. DiPalantino and Vojnovic [9] investigated the competition among crowdsourcing contests by proposing an interesting model. Moreover, the research on crowdsourcing contests is also analyzed by some papers. Yang et al. [43] found that the feedback offered by an employer can motivate workers to provide greater efforts using data from TaskCN.com. In another study, the case in which the rivalry and friendship in contests bring about an impact on a worker's chance of winning was considered by Mo et al. [23], who demonstrated that the triadic structures played a particularly significant role in the chance of winning. More recently, Huang et al. [15] proposed a dynamic structural model that illuminated the economic mechanisms shaping individual behavior and outcomes on such initiatives. Papanastasiou et al. [28] investigated the problem of optimal information provision when the goal was to maximize aggregate consumer surplus, which was motivated by the proliferation of online platforms. Allon and Babich [1] reviewed several key frameworks for analyzing crowdsourcing and believed that the fundamental ideas behind crowdsourcing had staying powers. Chen et al. [6] provided practical recommendations on setting up successful contests and attracting high-quality contestants to a contest on a crowdsourcing platform.

The second group of literature is the empirical research on the behaviors of workers. Liu et al. [21] investigated the impacts of the incentive mechanism on workers once they decided to undertake some crowdsourcing tasks and further implied that a higher reward tended to lead to a higher participation and higher quality answer based on the field experiment. Similar studies can also be found in Jeon et al. [16] and Chen et al. [7]. Later, there are also a few papers that mainly pay close attention to the mechanism design of a crowdsourcing platform. For example, Wen and Lin [41] established a game model of crowdsourcing services and further illustrated that the service fees of a platform were an increasing concave function of the task reward in the case of single or multiple contests. More recently, Jiang et al. [17] verified empirically that, with more conceptual objectives disclosed in the problem 
specification, the number of workers in a contest decreased. With two field data sets and an experimental study, Chan et al. [5] presented a comprehensive investigation of the complex effect of feedback on ideation performance in motivating crowdsourcing innovation. Ta et al. [37] examined the ways that variations in messages presented to crowdsourced agents could serve as a mechanism to enhance participation and associated performance outcomes.

Compared to the existing literature on crowdsourcing services aforementioned, our paper makes three significant contributions. First, our paper incorporates cooperative strategies among independent workers in the crowdsourcing service setting, and provides a unified and parsimonious model of cooperative game for studying workers' cooperative strategies. To the best of our knowledge, most literature on crowdsourcing services assumes that a given task is independently completed by individual workers. However, as the task becomes more diverse, employers often post some complex or difficult tasks that individual worker cannot complete. Thus, it is critical to analyze cooperative behaviors among independent workers to jointly complete a task in the crowdsourcing service setting. Second, our paper designs fair profit allocation policies for a workers coalition using the cooperative game theory and duality theory. A fair profit allocation policy not only motivates independent workers to cooperate but also ensures the stability of a workers coalition. Third, our paper analyzes a concave investment cost structure for workers and a convex quality reward structure for an employer in the crowdsourcing service setting. This allows us to find more results and management implications.

The third group of literature comprises studies that use methodologies similar to ours. In this paper, we propose a fair profit allocation policy for a workers coalition in crowdsourcing services. The notion of fairness is captured by the concept of core in the cooperative game theory [25]. It is well known that there are many theoretical studies on the cooperative game theory, such as Anily [2], Schlicher et al. [32], Chen and Zhang [8], Karsten et al. [20] and Schaarsberg et al. [33]. To propose a fair profit allocation policy, we present a duality approach to the capacity pooling game in crowdsourcing services, which is proposed by Owen [25], who proved that the core is nonempty for a class of linear production games using the duality theory of linear programs. The duality approach has been an important tool in analyzing the cooperative games and is widely applied in various fields [8, 38]. In this paper, we extend the duality approach to the capacity pooling game in crowdsourcing services and propose fair profit allocation policies for a workers coalition.

\section{Problem formulation}

\subsection{Cooperative games}

Prior to introducing the capacity pooling games in crowdsourcing services, we briefly give some basic concepts of the cooperative game theory that will be used in this paper; refer to Owen [26] and Peleg and Sudhölter [29] for more details. Denote by $N=\{1,2, \ldots, n\}$ a finite collection of players. Any subset $S \subseteq N$ is referred to as a coalition and the set of all possible coalitions is denoted by $2^{N}$. A coalition that 
comprises every player is called as the grand coalition $N$. A cooperative game is defined by a pair $(N, \pi)$, where $\pi: 2^{N} \rightarrow R$ is the value function. For any coalition $S \subseteq N$, the characteristic profit function $\pi(S)$ could be the maximum total profit that coalition $S$ should obtain if the players of coalition $S$ are to separate from the grand coalition and seek an optimal strategy for themselves. Moreover, the cooperative game $(S, \pi)$ for any subset $S \subseteq N$ is referred to as a subgame.

As is known to all, a central problem in the cooperative game theory is how to design a fair allocation policy to divide the profit (or cost) among all players of a coalition. However, it is noted that a poor allocation policy tends to result in distorted incentives and inefficient operations and even leads certain players to leave the current coalition [36]. A vector $l=\left(l_{1}, l_{2}, \ldots, l_{n}\right)$ is referred to as an allocation of the cooperative game $(N, \pi)$ if $\sum_{i \in N} l_{i}=\pi(N)$. In this paper, we particularly focus on the core allocation, which is defined below.

Definition 1 An allocation $l=\left(l_{1}, l_{2}, \ldots, l_{n}\right)$ is in the core of the cooperative game $(N, \pi)$, if

$$
\sum_{i \in N} l_{i}=\pi(N) \text { and } \sum_{i \in S} l_{i} \geq \pi(S), \forall S \subseteq N .
$$

The core is a basic solution concept in the cooperative game theory that requires that no group of payers would be better off by seceding from the cooperation. Consequently, designing a core profit allocation policy may create an incentive for players to cooperate. Nevertheless, judging whether the core of a cooperative game $(N, \pi)$ is nonempty or not is not straightforward. If yes, then there exists at least one profit allocation policy considered to be advantageous by all players of the coalition. We now give another significant concept of the balanced nature of a game.

Definition 2 A cooperative game $(N, \pi)$ is balanced, if

$$
\sum_{S \in 2^{N}} \tau(S) \pi(S) \leq \pi(N), \text { for any balanced map } \tau: 2^{N} \rightarrow[0,1] \text { and } \sum_{S \in 2^{N}} \tau(S) 1_{\{i \in S\}}=1,
$$

where $1_{\{i \in S\}}$ denotes the indicator function.

It is appropriate to point out that a balanced game always has a nonempty core but not every cooperative game is balanced [4]. More importantly, the balanced property ensures the stability of a coalition.

\subsection{The capacity pooling models in crowdsourcing services}

Consider a crowdsourcing service where an employer facing an unsolved problem posts his task online to a vast number of open workers. A reward budget is also provided by the employer to attract workers who are interested in this crowdsourcing task. In this paper, we mainly focus on a common scenario where the task posted online by an employer is relatively complex or difficult and thus individual workers cannot complete it independently. In this case, to obtain a reward provided by 
an employer, a group of independent workers may willingly form a workers coalition, denoted by $N=\{1,2, \ldots, n\}$, to jointly work on the crowdsourcing task in a collaborative way. That is to say, this crowdsourcing task would be divided into $n$ small pieces and every piece can be fulfilled independently by the corresponding worker $i$ in the workers coalition $N$ within a required time period.

For any workers coalition $S \subseteq N$, each worker $i \in S$ incurs a capacity investment $\operatorname{cost} c_{i}\left(q_{i}\right)$ for the investment level $q_{i}$. Denote by $Q$ the quality of an answer submitted by the workers coalition $S$, which is also referred to as the submitted quality. The employer pay a reward of $p(Q)$ depending on the submitted quality $Q$. Denote by $Q_{r}$ the realized quality of the submitted answer in a time period. It is appropriate to point out that the realized quality $Q_{r}$ of the submitted answer can be easily observed by the employer after the crowdsourcing transaction is completed. If the realized quality $Q_{r}$ becomes inconsistent with the submitted quality $Q$, the employer can ask for a refund of partial (or full) reward from the workers coalition $S$. Especially, the realized quality $Q_{r}$ may be zero in extreme cases. For example, an employer offers some strong evidences to illustrate that a submitted logo design is a plagiarism after the crowdsourcing transaction is over.

The crowdsourcing service mainly includes the bidding mode, the employment mode and the contrast mode (e.g., Zhubajie, TaskCN, DesignCrowd, InnoCentive). The sequence of events for the bidding mode in a crowdsourcing service happens as follows. First, an employer posts a complex or difficult task and a reward budget associated with this posted task on a crowdsourcing platform. Quite notably, as for the bidding mode, the reward provided by the employer can be modified at any time depending on the quality of the submitted answer until the end of the crowdsourcing service. Second, some workers coalitions interested in the crowdsourcing task posted by the employer begin to bid for this task, and then the employer choose one of the workers coalitions as the winning bidder to work on the posted task. Third, the winning workers coalition submits an answer of the task into the crowdsourcing platform. Fourth, the employer assesses the quality of the submitted answer and pay a reward depending on the submitted quality. Finally, in a time period, if the realized quality of the submitted answer observed by the employer becomes inconsistent with the submitted quality, the employer can ask for a refund of partial (or full) reward. In particular, the sequence of events for the employment mode is similar to that of the bidding mode, except that an employer relying on own demand of crowdsourcing task actively seeks for some workers coalitions and finally employs a proper workers coalition to complete own task. However, the sequence of events for the contrast mode is different from that of the bidding mode and the employment mode. In the contrast mode, each workers coalition interested in the task first submits a corresponding answer into the crowdsourcing platform, and then the employer chooses one or more among the submitted answers as the winner(s) and pays the prescribed reward.

It is important to point out that the contrast mode has three main characteristics; (i) In the contrast mode, the reward for the selected answer(s) is usually independently determined by the employer when releasing a task on the crowdsourcing platform and kept unchanged until the crowdsourcing service ends in general, which implies that the reward of the contrast mode may has nothing to do with the quality 
of the submitted answer; that is, the reward provided by an employer is a constant. Evidently, this reward structure differs from ones in the bidding and employment modes that are negotiated by both the employer and the workers coalition relying on the quality of the submitted answer; (ii) In the bidding mode, some workers coalitions bid for a certain crowdsourcing task simultaneously by providing preliminary schemes to solve this task. It is noted that the cost of designing a scheme for each workers coalition is very small, so that a failed bidder is considered to incur no cost. In the contrary, the losers in the contrast mode have to bear the costs of submitting their answers to the crowdsourcing task. What's more, this cost for completing the crowdsourcing task must not be ignored even if they lose the contrast; (iii) The losers, in the contrast mode, not only are not rewarded but also have to incur a cost of submitting answers. Thus, each player $i$ in the workers coalition $S \subseteq N$ is required to invest at least a minimum level, denoted by $\underline{q}$, to be a winner in the contrast mode.

As described above, considering whether the realized quality of the submitted answer $Q_{r}$ is consistent with the submitted quality $Q$, the workers coalition obtaining a reward may exist a refund or not. We first focus on the case in which there is no refund. For any workers coalition $S \subseteq N$ without a refund, the goal is to maximize the total profit of the workers coalition $S$, which includes the reward minus the capacity investment cost. The workers coalition makes the decisions on the capacity investment level of each player and the quality level of a submitted answer. Denote the maximum profit of the workers coalition $S$ without a refund by $\pi(S)$, which is the optimal objective value of the following optimization problem.

$$
\begin{aligned}
& \pi(S)=\max _{Q, q_{i}} p(Q)-\sum_{i \in S} c_{i}\left(q_{i}\right) \\
& \text { s.t. } q_{i} \leq q_{i}^{0}, i \in S, \\
& Q \leq \sum_{i \in S} q_{i}, \\
& Q \geq 0, q_{i} \geq 0,
\end{aligned}
$$

where the upper bound $q_{i}^{0}$ denotes the nameplate capacity of each worker $i \in S$. The first constraint shows that every individual investment level is no more than his nameplate capacity and the second constraint is the capacity constraint. The cooperative capacity pooling game without a refund in a crowdsourcing service is the pair $(N, \pi)$, where the characteristic profit function $\pi$ is given by problem (1).

Next, we pay attention to another case in which the workers coalition is required a refund. It is noted that the workers coalition with a refund is responsible for not only the capacity investment cost but also the refund cost. Denote by $\pi_{r}(S)$, for any workers coalition $S \subseteq N$ with a refund, the maximum profit of the workers coalition $S$, which is defined as follows: 


$$
\begin{aligned}
& \pi_{r}(S)=\max _{Q, q_{i}} p(Q)-\sum_{i \in S} c_{i}\left(q_{i}\right)-\varphi\left(Q-Q_{r}\right)^{+} \\
& \text {s.t. } \quad q_{i} \leq q_{i}^{0}, \quad i \in S, \\
& Q \leq \sum_{i \in S} q_{i}, \\
& Q \geq 0, q_{i} \geq 0,
\end{aligned}
$$

where $x^{+}:=\max \{x, 0\}$ for all $x \in R$, the term $\varphi\left(Q-Q_{r}\right)^{+}$implies the refund and $\varphi$ is unit refund cost. The cooperative capacity pooling game with a refund is the pair $\left(N, \pi_{r}\right)$, where the characteristic profit function $\pi_{r}$ is defined by problem (2).

Remark 1 When $\varphi\left(Q-Q_{r}\right)^{+}=0$, i.e., $\varphi=0$ or $Q_{r}=Q$, in problem (2), it is easy to verify that problem (2) is reduced to problem (1). In other words, problem (1) is a special case of problem (2). For this reason, we will fist analyze the capacity pooling game with a refund defined by problem (2). Then, in a similar way, the results of the capacity pooling game without a refund defined by problem (1) can be directly gained.

Remark 2 To avoid some trivial cases, we assume that $\varphi\left(Q-Q_{r}\right)^{+} \leq p(Q)$ in problem (2), which shows that the refund from the workers coalition is no more than their obtained reward. This assumption is vary appropriate in practice. Moreover, in this paper, we mainly focus on the case of $p(Q)>0$ in problems (1) and (2), which illustrates that the workers coalition $S$ is considered as a winning bidder in the bid mode, an employee in the employment mode or a winner in the contrast mode. On the other hand, if $p(Q)=0$, the workers coalition $S$ loses the opportunity of working on the corresponding crowdsourcing task in the bid mode and employment mode. However, in the contrast mode, the workers coalition $S$ has submitted an answer to solve this task, but it is not selected by the employer in the end.

\section{Capacity pooling game in the bidding mode}

In this section, we analyze the capacity pooling game in the bidding mode. We remark that the bidding mode has been one of the most popular modes in crowdsourcing services and is becoming increasingly popular. Generally speaking, this bidding mode is suitable for some complex types of tasks, such as software development, house decoration, scientific research, video production and website construction.

\subsection{Linear investment cost and quality reward}

In this subsection, we focus on the case where the capacity investment cost function of each worker $i$ in a workers coalition $S \subseteq N$ and the quality reward function set by an employer are both assumed to be linear. By abusing notations, we denote $c_{i}\left(q_{i}\right)=c_{i} q_{i}$ and $p(Q)=p Q$, where $c_{i}$ and $p$ denote the unit investment cost 
and unit quality reward, respectively. This assumption is appropriate for settings in which the economies of scale are negligible and is widely adopted in reality. More interestingly, this subsection serves as a basis for the analyses in the next subsections. In this case, for any workers coalition $S \subseteq N$ with a refund, problem (2) becomes a linear programming problem, which is given by

$$
\begin{aligned}
& \pi_{r}(S)=\max _{Q, q_{i}} p Q-\sum_{i \in S} c_{i} q_{i}-\varphi\left(Q-Q_{r}\right)^{+} \\
& \text {s.t. } \quad q_{i} \leq q_{i}^{0}, i \in S, \\
& Q \leq \sum_{i \in S} q_{i}, \\
& Q \geq 0, q_{i} \geq 0 .
\end{aligned}
$$

For ease of analysis, we introduce a variable $d$ so that problem (3) is reformulated equivalently as:

$$
\begin{aligned}
& -\pi_{r}(S)=\min _{Q, q_{i}, d}-p Q+\sum_{i \in S} c_{i} q_{i}+\varphi d \\
& \text { s.t. } \quad q_{i} \leq q_{i}^{0}, \quad i \in S, \\
& Q-\sum_{i \in S} q_{i} \leq 0, \\
& Q-d \leq Q_{r}, \\
& Q \geq 0, q_{i} \geq 0, d \geq 0,
\end{aligned}
$$

where $d$ denotes the lost quality of the workers coalition $S$. We now apply the linear programming duality approach to the primal problem (4). Immediately, the duality result of (4) is derived

$$
\begin{aligned}
& \max _{\lambda_{i}, \alpha, \beta} \sum_{i \in S} \lambda_{i} q_{i}^{0}+\beta Q_{r} \\
& \text { s.t. } \quad \lambda_{i}-\alpha \leq c_{i}, \quad i \in S, \\
& \alpha+\beta \leq-p, \quad-\beta \leq \varphi, \\
& \lambda_{i} \leq 0, \quad \alpha \leq 0, \quad \beta \leq 0,
\end{aligned}
$$

where $\lambda_{i}, \alpha$ and $\beta$ are the dual variables associated with the first, second and third constraints in (4), respectively.

Lemma 1 For any collection of workers $S \subseteq N$ with a refund in a crowdsourcing service, $-\pi_{r}(S)$ defined by (4) is equal to the optimal value of (5).

Lemma 1 illustrates the strong duality theory; that is, $-\pi_{r}(S)$ is equal to the optimal value of (5), which plays a particularly significant role in designing a fair allocation policy in this study. Denote by $\left(Q^{*}, q^{*}, d^{*}\right)$ an optimal solution to the primal problem (4), and $\left(\lambda^{*}, \alpha^{*}, \beta^{*}\right)$ an optimal dual solution to the dual problem (5). 
As discussed previously, for the workers coalition $S \subseteq N$ with a refund, the realized quality of the submitted answer is less than the submitted quality, i.e., $Q_{r}<Q$. In this case, the closed-form solutions of these decision variables are gained using the corresponding complementary slackness conditions.

Proposition 1 When there exists a refund, these optimal solutions $Q^{*}, q^{*}, d^{*}, \lambda^{*}, \alpha^{*}$ and $\beta^{*}$ are given by.

$$
q_{i}^{*}=q_{i}^{0}, \quad Q^{*}=\sum_{i \in S} q_{i}^{0}, \quad \lambda_{i}^{*}=\varphi-p+c_{i}, \quad \alpha^{*}=\varphi-p, \quad \beta^{*}=-\varphi \text { and } d^{*}=\sum_{i \in S} q_{i}^{0}-Q_{r} .
$$

According to Proposition 1, we know that, in order to maximize their own utilities, each player of the workers coalition $S$ has to try their best to accomplish the crowdsourcing task for which they are responsible. In other words, each worker $i \in S$ uses his or her nameplate capacity to undertake the corresponding crowdsourcing task, i.e., $q_{i}^{*}=q_{i}^{0}$. In the meantime, the workers coalition $S$ also has to submit a highest quality answer to the crowdsourcing task into the crowdsourcing platform within their capabilities, i.e., $Q^{*}=\sum_{i \in S} q_{i}^{0}$. Additionally, it is obvious to verify that the optimal dual solution $\left(\lambda^{*}, \alpha^{*}, \beta^{*}\right)$ depends on the unit refund cost $\varphi$, unit investment cost $c_{i}$ and unit quality reward $p$, which, in particular, plays an important role in designing a fair profit allocation policy for the workers coalition $S$ with a refund. We are now ready to propose a profit allocation policy among players of the workers coalition and prove the proposed allocation policy in the core of the capacity pooling game $\left(N, \pi_{r}\right)$.

Recall that $\left(\lambda^{*}, \alpha^{*}, \beta^{*}\right)$ is an optimal solution to the dual problem (5) with $S=N$. Define for any $i \in N$,

$$
l_{r i}=-\left(\lambda_{i}^{*} q_{i}^{0}+\frac{\beta^{*} Q_{r}}{n}\right)
$$

Proposition 2 The vector $l_{r}=\left(l_{r 1}, l_{r 2}, \ldots, l_{r n}\right)$ defined by (6) is a profit allocation policy in the core of the capacity pooling game $\left(N, \pi_{r}\right)$.

Proposition 2 shows the non-emptiness of the core of the capacity pooling game $\left(N, \pi_{r}\right)$ by providing a constructive proof. That is to say, the profit allocation policy $l_{r}=\left(l_{r 1}, l_{r 2}, \ldots, l_{r n}\right)$ defined by (6) is fair for players of the workers coalition $N$ with a refund. The fair profit allocation policy motivates independent workers to willingly form a coalition to jointly undertake a crowdsourcing task, and thus ensures the stability of the workers coalition. More interestingly, Proposition 2 proposes a simple way to compute a fair profit allocation policy for the workers coalition with a refund by solving the dual problem of a linear program. Namely, a fair profit allocation policy can be defined by any given optimal dual solution. Thus, Proposition 2 provides a unified and parsimonious framework of fair profit allocation policy for studying the workers' capacity pooling when faced with a complex or difficult task in crowdsourcing services. Furthermore, we have $l_{r i}=\left(p-\varphi-c_{i}\right) q_{i}^{0}+\varphi Q_{r} / n$. Therefore, the unit refund cost, investment cost and 
quality reward play a significant role in the proposed fair profit allocation policy, which is analyzed in detail in Sect. 6.

Corollary 1 The resulting capacity pooling game $\left(N, \pi_{r}\right)$ is balanced.

The balance of the capacity pooling game $\left(N, \pi_{r}\right)$ guarantees that there exists at least one fair profit allocation policy for the workers coalition $N$ with a refund. In other words, the balance ensures the stability of the workers coalition $N$ with a refund. Next, we turn our attention to another case. For any workers coalition $S \subseteq N$ without a refund, problem (1) also becomes a linear programming problem.

$$
\begin{aligned}
& \pi(S)=\max _{Q, q_{i}} p Q-\sum_{i \in S} c_{i} q_{i} \\
& \text { s.t. } \quad q_{i} \leq q_{i}^{0}, i \in S, \\
& Q \leq \sum_{i \in S} q_{i}, \\
& Q \geq 0, q_{i} \geq 0 .
\end{aligned}
$$

The dual of problem (7) is directly obtained that

$$
\begin{aligned}
& \min _{\lambda_{i}, \alpha} \sum_{i \in S} \lambda_{i} q_{i}^{0} \\
& \text { s.t. } \quad \lambda_{i}-\alpha \geq-c_{i}, \quad i \in S, \\
& \alpha \geq p, \\
& \lambda_{i} \geq 0, \quad \alpha \geq 0,
\end{aligned}
$$

where $\lambda_{i}$ and $\alpha$ are the dual variables associated with the first and second constraints in problem (7), respectively.

Lemma 2 For any workers coalition $S \subseteq N$ without a refund in a crowdsourcing service, $\pi(S)$ defined by (7) is equal to the optimal value of (8).

Denote by $\left(Q^{*}, q^{*}\right)$ an optimal solution to the primal problem (7), and $\left(\lambda^{*}, \alpha^{*}\right)$ an optimal dual solution to the dual problem (8). For the workers coalition $S \subseteq N$ without a refund, the realized quality of an answer submitted by the workers coalition $S$ is consistent with the submitted quality, i.e., $Q_{r}=Q$. In this case, we also gain the closed-form solutions of these decision variables.

Proposition 3 When there is no refund, these optimal solutions $Q^{*}, q^{*}, \lambda^{*}$ and $\alpha^{*}$ is given by

$$
q_{i}^{*}=q_{i}^{0}, Q^{*}=\sum_{i \in S} q_{i}^{0}, \lambda_{i}^{*}=p-c_{i} \text { and } \alpha^{*}=p
$$

When there is no refund, Proposition 3 implies that, to get a better reward, each player of the workers coalition should try their best to work on the crowdsourcing 
task, i.e., $q_{i}^{*}=q_{i}^{0}$. Moreover, the workers coalition should submit a highest quality answer within their capacities to the employer, i.e., $Q^{*}=\sum_{i \in S} q_{i}^{0}$. In addition, we observe that the optimal dual solution $\left(\lambda^{*}, \alpha^{*}\right)$ is associated with the unit investment cost $c_{i}$ and unit quality reward $p$, which lays an important foundation in the design of a fair profit allocation policy for the workers coalition $S$ without a refund. We summarize the results from Proposition 1 and Proposition 3, and achieve an interesting managerial insight.

Corollary 2 Whether there is a refund or not in crowdsourcing services, a workers coalition has to try their best to work on the crowdsourcing task and submit an answer of the highest quality.

Next, we prepare to give an allocation policy for the workers coalition without a refund based on the duality theory. Recall that $\left(\lambda^{*}, \alpha^{*}\right)$ is an optimal solution to the dual problem (8) with $S=N$. Define

$$
l_{i}=\lambda_{i}^{*} q_{i}^{0}, \quad i \in N .
$$

Proposition 4 The vector $l=\left(l_{1}, l_{2}, \ldots, l_{n}\right)$ defined by (9) is a profit allocation policy in the core of the capacity pooling game $(N, \pi)$.

When there is no refund, Proposition 4 provides a constructive proof to the non-emptiness of the core of the capacity pooling game $(N, \pi)$. Namely, the vector $l=\left(l_{1}, l_{2}, \ldots, l_{n}\right)$ defined by (9) is a fair profit allocation policy for the workers coalition $N$ without a refund. Meanwhile, for any player $i \in N$, we know that the allocated profit is $l_{i}=\left(p-c_{i}\right) q_{i}^{0}$. Thus, both the unit quality reward and investment cost play a significant role in the proposed fair profit allocation policy for the workers coalition without a refund, which is given a detailed analysis in Sect. 6. We now provide some intuitions on the two fair profit allocation policies proposed by Proposition 2 and Proposition 4. When there is no refund, the allocated profit defined by (9) for each worker $i \in N$ mainly depends on the term of $\lambda_{i}^{*} q_{i}^{0}$, a similar term is also included in (6). However, the allocated profit defined by (6) for each worker $i \in N$ facing a refund also depends on the term of $\beta^{*} Q_{r} / n$, which implies that the refund is paid equally by each player of the workers coalition.

\subsection{Concave investment cost}

In this subsection, we are interested in the capacity pooling games $\left(N, \pi_{r}\right)$ and $(N, \pi)$ with a concave investment cost structure, respectively. We assume that the capacity investment cost of each player in a workers coalition is a concave function of investment level, which exhibits the economies of scale. That is to say, the higher the investment level, the lower the average unit investment cost. We would like to point out that the concave investment cost structure has been widely applied in practice [8]. 
For each player $i$ in the workers coalition $N$, denote by $c_{i}(\cdot)$ a concave capacity investment cost function of investment level. Without loss of generality, the concave investment cost function $c_{i}(\cdot)$ is assumed continuously differentiable. The capacity pooling games analyzed in this subsection are exactly the same as ones considered in Sect. 4.1, except for the concave investment cost of each worker.

We first focus on the case in which there is a refund, for any workers coalition $S \subseteq N$, the characteristic profit function $\pi_{r}(S)$ with a concave investment cost structure is defined as

$$
\begin{aligned}
& \pi_{r}(S)=\max _{Q, q_{i}} p Q-\sum_{i \in S} c_{i}\left(q_{i}\right)-\varphi\left(Q-Q_{r}\right)^{+} \\
& \text {s.t. } \quad q_{i} \leq q_{i}^{0}, i \in S, \\
& Q \leq \sum_{i \in S} q_{i}, \\
& Q \geq 0, q_{i} \geq 0 .
\end{aligned}
$$

Furthermore, we introduce a variable $d$ so that problem (10) is reformulated as a two-stage programming problem.

$$
\begin{aligned}
& -\pi_{r}(S)=\min _{q_{i}} \sum_{i \in S} c_{i}\left(q_{i}\right)+f(q) \\
& \text { s.t. } \quad q_{i} \leq q_{i}^{0}, \quad i \in S,
\end{aligned}
$$

where $q=\left(q_{i}\right)_{i \in S}$ and $f(q)$ is defined by

$$
\begin{aligned}
& f(q)=\min _{Q, d}-p Q+\varphi d \\
& \text { s.t. } \quad Q-\sum_{i \in S} q_{i} \leq 0 \\
& Q-d \leq Q_{r} \\
& Q \geq 0, q_{i} \geq 0, d \geq 0 .
\end{aligned}
$$

Since the investment cost $c_{i}\left(q_{i}\right)$ included in problem (11) is a concave function, it poses a huge challenge to design a fair profit allocation policy. For this purpose, we need to introduce a significant result.

Lemma 3 Assume $F$ is a concave function on a convex neighborhood $D \subset R^{n}$ of $x$, and differentiable at $x$. Then,

$$
F(x)+F^{\prime}(x)(y-x) \geq F(y), \text { for every } y \text { in } D .
$$

Define the function $H_{x}$ by $H_{x}(y)=F(x)+F^{\prime}(x)(y-x)$ for any $x \in D$. Therefore, by Lemma 3, we know that $H_{x}$ is a concave function, $F \leq H_{x}$ for each $x$ and $F(x)=H_{x}(x)$. Immediately, a significant result is derived that $F=\min _{x \in D} H_{x}$; that is,

$$
F(y)=\min _{x \in D} F(x)+F^{\prime}(x)(y-x) .
$$


After obtaining the important result given by Eq. (12), we now pay close attention to solving the duality result of the characteristic profit function $\pi_{r}(S)$ with a concave investment cost structure defined by (11). For any workers coalition $S \subseteq N$, define

$$
\Theta^{*}=\min _{x_{i} \in \Delta} \sum_{i \in S} c_{i}\left(x_{i}\right)+f(x)
$$

where $c_{i}(\cdot)$ is a concave function with $c_{i}(0)=0$ and $\Delta=\left\{q_{i} \mid q_{i} \leq q_{i}^{0}, i \in S\right\}$. Denote by $\nabla c_{i}(\cdot)$ a supergradient of the concave function $c_{i}(\cdot)$. Thus, we have

$$
c_{i}\left(x_{i}\right)=\min _{q_{i} \in \Delta} c_{i}\left(q_{i}\right)+\nabla c_{i}\left(q_{i}\right)\left(x_{i}-q_{i}\right) .
$$

Substituting $c_{i}\left(x_{i}\right)$ given by Eq. (14) into Eq. (13), we gain that

$$
\begin{aligned}
\Theta^{*} & =\min _{x_{i} \in \Delta} \min _{q_{i} \in \Delta} \sum_{i \in S} c_{i}\left(q_{i}\right)-\nabla c_{i}\left(q_{i}\right) q_{i}+\nabla c_{i}\left(q_{i}\right) x_{i}+f(x) \\
& =\min _{q_{i} \in \Delta} \sum_{i \in S} c_{i}\left(q_{i}\right)-\nabla c_{i}\left(q_{i}\right) q_{i}+\min _{x_{i} \in \Delta} \sum_{i \in S} \nabla c_{i}\left(q_{i}\right) x_{i}+f(x) .
\end{aligned}
$$

Consequently, we have

$$
\begin{aligned}
& \Theta^{*}=\max \Theta \\
& \text { s.t. } \quad \Theta \leq \sum_{i \in S} c_{i}\left(q_{i}\right)-\nabla c_{i}\left(q_{i}\right) q_{i}+\min _{x_{i} \in \Delta} \sum_{i \in S} \nabla c_{i}\left(q_{i}\right) x_{i}+f(x), \forall q_{i} \in \Delta .
\end{aligned}
$$

Moreover, it is noted that for any given $q_{i}$, the minimization problem

$$
\min _{x_{i} \in \Delta} \sum_{i \in S} \nabla c_{i}\left(q_{i}\right) x_{i}+f(x)
$$

is a two-stage linear programming problem, whose duality result is given by

$$
\begin{aligned}
& \max _{t_{i}, \alpha, \beta} \sum_{i \in S} t_{i} q_{i}^{0}+\beta Q_{r} \\
& \text { s.t. } \quad t_{i}-\alpha \leq \nabla c_{i}\left(q_{i}\right), \quad i \in S, \\
& \alpha+\beta \leq-p, \quad-\beta \leq \varphi, \\
& t_{i} \leq 0, \quad \alpha \leq 0, \quad \beta \leq 0 .
\end{aligned}
$$

Therefore, based on the duality result given by problem (15), we have

$$
\Theta^{*}=\max \Theta
$$

$$
\begin{aligned}
& \text { s.t. } \Theta \leq \sum_{i \in S} c_{i}\left(q_{i}\right)-\nabla c_{i}\left(q_{i}\right) q_{i}+\sum_{i \in S} t_{i} q_{i}^{0}+\beta Q_{r}, \forall q_{i} \in \Delta, \\
& t_{i}-\alpha \leq \nabla c_{i}\left(q_{i}\right), i \in S, \forall q_{i} \in \Delta, \\
& \alpha+\beta \leq-p,-\beta \leq \varphi, \\
& t_{i} \leq 0, \alpha \leq 0, \beta \leq 0
\end{aligned}
$$


Moreover, one can define variable $\rho_{i}$ for any given $q_{i}$ so that

$$
\sum_{i \in S} c_{i}\left(q_{i}\right)-\nabla c_{i}\left(q_{i}\right) q_{i} \geq \sum_{i \in S} \rho_{i} q_{i}^{0} .
$$

We further introduce variable $\lambda_{i}$ such that $\lambda_{i} \leq \rho_{i}+t_{i}$. Consequently, the duality result of the two-stage programming problem (11) is obtained that

$$
\begin{aligned}
& \max \quad \sum_{i \in S} \lambda_{i} q_{i}^{0}+\beta Q_{r} \\
& \text { s.t. } \quad \lambda_{i} \leq \rho_{i}+t_{i}, i \in S, \\
& \sum_{i \in S} \rho_{i} q_{i}^{0} \leq \sum_{i \in S} c_{i}\left(q_{i}\right)-\nabla c_{i}\left(q_{i}\right) q_{i}, \forall q_{i} \in \Delta, \\
& t_{i}-\alpha \leq \nabla c_{i}\left(q_{i}\right), i \in S, \forall q_{i} \in \Delta, \\
& \alpha+\beta \leq-p, \quad-\beta \leq \varphi, \\
& t_{i} \leq 0, \alpha \leq 0, \beta \leq 0,
\end{aligned}
$$

where $\lambda_{i}, \rho_{i}, t_{i}, \alpha$ and $\beta$ are the dual variables. Additionally, the duality result given by problem (16) is similar to the one for problem (5) except for the concave capacity investment cost $c_{i}\left(q_{i}\right)$ of each worker $i \in S$. When the investment level of each player $i$ in the workers coalition $S$ is $q_{i}$, the incurred cost $c_{i}\left(q_{i}\right)$ is divided into a proportional cost $\nabla c_{i}\left(q_{i}\right)$ and a fixed cost $c_{i}\left(q_{i}\right)-\nabla c_{i}\left(q_{i}\right) q_{i}$, which is different from the case in which the capacity investment cost function is linear. Next, using the duality theory, we propose a profit allocation policy and prove the proposed allocation policy in the core of the capacity pooling game $\left(N, \pi_{r}\right)$ with a concave investment cost.

Denote by $\left(\lambda^{*}, \rho^{*}, t^{*}, \alpha^{*}, \beta^{*}\right)$ an optimal solution to the dual problem (16) with $S=N$. Define for any $i \in N$,

$$
l_{r i}=-\left(\lambda_{i}^{*} q_{i}^{0}+\frac{\beta^{*} Q_{r}}{n}\right)
$$

Proposition 5 The vector $l_{r}=\left(l_{r 1}, l_{r 2}, \ldots, l_{r n}\right)$ defined by Eq. (17) is a profit allocation policy in the core of the capacity pooling game $\left(N, \pi_{r}\right)$ with a concave investment cost structure, if $\rho^{*} \leq 0$.

Proposition 5 illustrates that the profit allocation policy defined by Eq. (17) for each player of the workers coalition with a refund, facing the economies of scale, is fair. The existence of a fair profit allocation policy provides an incentive for independent workers to cooperate to jointly undertake a crowdsourcing task by pooling their capacities together, with the goal of obtaining a reward from an employer. When faced with the economies of scale, Proposition 5 provides a duality approach to design a fair profit allocation policy for the workers coalition with a refund. We would like to point out that the duality approach used in this paper is always effective in designing a fair allocation policy, at least for problems of moderate sizes, by solving a linear program. Notably, Proposition 5 requires the condition that $\rho^{*} \leq 0$, 
which guarantees that the profit allocation policy $l_{r}=\left(l_{r 1}, l_{r 2}, \ldots, l_{r n}\right)$ defined by Eq. (17) is fair.

Next, we investigate another case where there is no refund. For any workers coalition $S \subseteq N$, the characteristic profit function $\pi(S)$ with a concave investment cost is given by

$$
\begin{aligned}
& \pi(S)=\max _{Q, q_{i}} p Q-\sum_{i \in S} c_{i}\left(q_{i}\right) \\
& \text { s.t. } \quad q_{i} \leq q_{i}^{0}, \quad i \in S, \\
& Q \leq \sum_{i \in S} q_{i}, \\
& Q \geq 0, q_{i} \geq 0 .
\end{aligned}
$$

In a similar way, we gain the duality result of problem (18) immediately, which is given as follows:

$$
\begin{aligned}
& \min -\sum_{i \in S} \lambda_{i} q_{i}^{0} \\
& \text { s.t. } \quad \lambda_{i} \leq \rho_{i}+t_{i}, \quad i \in S, \\
& \sum_{i \in S} \rho_{i} q_{i}^{0} \leq \sum_{i \in S} c_{i}\left(q_{i}\right)-\nabla c_{i}\left(q_{i}\right) q_{i}, \forall q_{i} \in \Delta, \\
& t_{i}-\alpha \leq \nabla c_{i}\left(q_{i}\right), \quad i \in S, \forall q_{i} \in \Delta, \\
& \alpha \leq-p, \\
& t_{i} \leq 0, \alpha \leq 0,
\end{aligned}
$$

where $\lambda_{i}, \rho_{i}, t_{i}$ and $\alpha$ are the dual variables of problem (19). Denote by $\left(\lambda^{*}, \rho^{*}, t^{*}, \alpha^{*}\right)$ an optimal solution to the dual problem (19) with $S=N$. Define

$$
l_{i}=-\lambda_{i}^{*} q_{i}^{0}, \quad i \in N
$$

Proposition 6 The vector $l=\left(l_{1}, l_{2}, \ldots, l_{n}\right)$ defined by Eq. (20) is a profit allocation policy in the core of the capacity pooling game $(N, \pi)$ with a concave investment cost, if $\rho^{*} \leq 0$.

Facing the economies of scale, Proposition 6 not only demonstrates that the core of the capacity pooling game $(N, \pi)$ with a concave investment cost is nonempty, but also gives a fair profit allocation policy for the workers coalition without a refund using the duality theory. The condition that $\rho^{*} \leq 0$, required in Proposition 6 , ensures that the profit allocation policy $l=\left(l_{1}, l_{2}, \ldots, l_{n}\right)$ defined by (20) under no refund is fair.

\subsection{Convex quality reward}

In this subsection, we focus on another case that motivates independent workers to form a workers coalition and jointly submit a high quality answer to an 
employer. We assume that the quality reward set by an employer is a convex function of the quality level. In other words, the higher the quality level, the higher the average unit quality reward. Interestingly, the setting of a convex quality reward is widely applied in reality. For example, it arises when the suppliers provide incremental incentives to additional units beyond some threshold to encourage their workers to submit a higher quality result. Denote by $p(\cdot)$ a convex quality reward function of the quality level submitted by a workers coalition. Meanwhile, the convex quality reward function $p(\cdot)$ is assumed continuously differentiable for the sake of analysis. We remark that the capacity pooling games investigated in this subsection are exactly the same as ones analyzed in Sect. 4.1, except for the convex quality reward structure.

We now focus on the first case in which there is a refund. Thus, the characteristic profit function $\pi_{r}(S)$ with a convex quality reward, for any workers coalition $S \subseteq N$, is given by

$$
\begin{aligned}
& \pi_{r}(S)=\max _{Q, q_{i}} p(Q)-\sum_{i \in S} c_{i} q_{i}-\varphi\left(Q-Q_{r}\right)^{+} \\
& \text {s.t. } \quad q_{i} \leq q_{i}^{0}, i \in S, \\
& Q \leq \sum_{i \in S} q_{i}, \\
& Q \geq 0, q_{i} \geq 0 .
\end{aligned}
$$

We further formulate problem (21) as a two-stage programming problem:

$$
\begin{aligned}
& \pi_{r}(S)=\max _{Q} p(Q)+g(Q) \\
& \text { s.t. } \quad Q \geq 0,
\end{aligned}
$$

where $g(Q)$ is given by

$$
\begin{aligned}
& g(Q)=\max _{q_{i}}-\sum_{i \in S} c_{i} q_{i}-\varphi\left(Q-Q_{r}\right)^{+} \\
& \text {s.t. } \quad q_{i} \leq q_{i}^{0}, i \in S, \\
& Q \leq \sum_{i \in S} q_{i}, q_{i} \geq 0 .
\end{aligned}
$$

As described above, the quality reward function $p(Q)$ set by an employer is convex, so it brings about a huge challenge in solving the duality result of the two-stage programming problem (22). For this purpose, we present an important result to simplify the corresponding convex structure.

Lemma 4 Assume $G$ is a convex function on a convex neighborhood $C \subset R^{n}$ of $x$, and differentiable at $x$. Then, it satisfies.

$$
G(y) \geq G(x)+G^{\prime}(x)(y-x) \text { for any } y \in C .
$$


By Lemma 4, define the function $J_{x}$ by $J_{x}(y)=G(x)+G^{\prime}(x)(y-x)$ for each $x$ in $C$, we know that $J_{x}$ is a convex function, $G \geq J_{x}$ for $x \in C$ and $G(x)=J_{x}(x)$ immediately. Therefore, we have $G=\max _{x \in C} J_{x}$; that is,

$$
G(y)=\max _{x \in C} G(x)+G^{\prime}(x)(y-x) .
$$

We now turn our attention to solving the dual problem of the two-stage programming problem (22). Denote

$$
\Phi^{*}=\max _{y \geq 0} p(y)+g(y),
$$

where $p(\cdot)$ is a convex function with $p(0)=0$. Denote by $\nabla p(\cdot)$ a subgradient of the convex function $p(\cdot)$. Hence, it follows from Lemma Lemma 4 that

$$
p(y)=\max _{Q \geq 0} p(Q)+\nabla p(Q)(y-Q) .
$$

Then, replacing $p(y)$ in (23) with the equation given by (24), we have

$$
\begin{aligned}
\Phi^{*} & =\max _{y \geq 0} \max _{Q \geq 0} p(Q)-\nabla p(Q) Q+\nabla p(Q) y+g(y) \\
& =\max _{Q \geq 0} p(Q)-\nabla p(Q) Q+\max _{y \geq 0} \nabla p(Q) y+g(y) .
\end{aligned}
$$

As a result, we gain directly that

$$
\begin{aligned}
& \Phi^{*}=\min \Phi \\
& \text { s.t. } \Phi \geq p(Q)-\nabla p(Q) Q+\max _{y \geq 0} \nabla p(Q) y+g(y), \forall Q \geq 0 .
\end{aligned}
$$

It is noted that, for any given $Q$, the maximization problem

$$
\max _{y \geq 0} \nabla p(Q) y+g(y)
$$

is a two-stage programming problem. We introduce a variable $d$ so that the dual of (26) is obtained immediately that

$$
\begin{aligned}
& \min \sum_{i \in S} u_{i} q_{i}^{0}+\beta Q_{r} \\
& \text { s.t. } \quad-u_{i}+\alpha \leq c_{i}, \quad i \in S . \\
& -\alpha-\beta \leq-\nabla p(Q), \quad \beta \leq \varphi, \\
& u_{i} \geq 0, \alpha \geq 0, \quad \beta \geq 0,
\end{aligned}
$$

where $u_{i}, \alpha$ and $\beta$ are the dual variables. Consequently, it follows directly from Lemma 1 that 


$$
\begin{aligned}
& \Phi^{*}=\min \Phi \\
& \text { s.t. } \quad \Phi \geq p(Q)-\nabla p(Q) Q+\sum_{i \in S} u_{i} q_{i}^{0}+\beta Q_{r}, \forall Q \geq 0 . \\
& -u_{i}+\alpha \leq c_{i}, \quad i \in S . \\
& -\alpha-\beta \leq-\nabla p(Q), \forall Q \geq 0, \beta \leq \varphi, \\
& u_{i} \geq 0, \alpha \geq 0, \beta \geq 0 .
\end{aligned}
$$

Moreover, one can define the variable $\eta_{i}$ so that

$$
p(Q)-\nabla p(Q) Q \leq \sum_{i \in S} \eta_{i} q_{i}^{0} \text { for any given } Q .
$$

We further define variable $\lambda_{i}$ so that $\lambda_{i} \geq \eta_{i}+u_{i}$. Hence, the dual of the two-stage programming problem (22) is obtained that

$$
\begin{aligned}
& \min \quad \sum_{i \in S} \lambda_{i} q_{i}^{0}+\beta Q_{r} \\
& \text { s.t. } \quad \lambda_{i} \geq \eta_{i}+u_{i}, \quad i \in S, \\
& p(Q)-\nabla p(Q) Q \leq \sum_{i \in S} \eta_{i} q_{i}^{0}, \quad \forall Q \geq 0, \\
& -u_{i}+\alpha \leq c_{i}, \quad i \in S, \\
& -\alpha-\beta \leq-\nabla p(Q), \quad \forall Q \geq 0, \beta \leq \varphi, \\
& u_{i} \geq 0, \quad \alpha \geq 0, \quad \beta \geq 0,
\end{aligned}
$$

where $\lambda_{i}, \eta_{i}, u_{i}, \alpha$ and $\beta$ are the variables of the dual problem (27). Next, based on the duality result given by problem (27), we prepare to propose a fair allocation policy for players in a workers coalition facing a convex quality reward.

Denote by $\left(\lambda^{*}, \eta^{*}, u^{*}, \alpha^{*}, \beta^{*}\right)$ an optimal solution to the dual problem (27) with $S=N$. Define for any $i \in N$.

$$
l_{r i}=\lambda_{i}^{*} q_{i}^{0}+\frac{\beta^{*} Q_{r}}{n}
$$

Proposition 7 The vector $l_{r}=\left(l_{r 1}, l_{r 2}, \ldots, l_{r n}\right)$ defined by Eq. (28) is a profit allocation policy in the core of the capacity pooling game $\left(N, \pi_{r}\right)$ with a convex quality reward structure, if $\eta^{*} \geq 0$.

When an employer set a convex quality reward structure, Proposition 7 uses a duality approach to illustrate that the proposed profit allocation policy $l_{r}=\left(l_{r 1}, l_{r 2}, \ldots, l_{r n}\right)$ defined by (28) under a refund is fair, which motivates independent workers to cooperate and jointly complete a crowdsourcing task. Nevertheless, it is obvious to observe that Proposition 7 requires the condition that $\eta^{*} \geq 0$, which plays a particularly significant role in constructing a fair profit allocation policy. In other words, the condition assures that any optimal dual solution to the grand workers coalition $N$ is feasible to the dual problem with $S \subseteq N$. Furthermore, Proposition 7 also shows that there exists 
at least one allocation policy in the core of the capacity pooling game $\left(N, \pi_{r}\right)$ with a convex quality reward structure, so the resulting capacity pooling game $\left(N, \pi_{r}\right)$ with a convex quality reward is balanced [4].

Next, we analyze another case where there is no refund. For any workers coalition $S \subseteq N$ without a refund, the characteristic profit function $\pi(S)$ with a convex quality reward is given by

$$
\begin{aligned}
& \pi(S)=\max _{Q, q_{i}} p(Q)-\sum_{i \in S} c_{i} q_{i} \\
& \text { s.t. } \quad q_{i} \leq q_{i}^{0}, \quad i \in S, \\
& Q \leq \sum_{i \in S} q_{i}, \\
& Q \geq 0, q_{i} \geq 0 .
\end{aligned}
$$

Following a similar way, the dual problem of problem (29) is obtained directly.

$$
\begin{array}{ll}
\min & \sum_{i \in S} \lambda_{i} q_{i}^{0} \\
\text { s.t. } \quad \lambda_{i} \geq \eta_{i}+u_{i}, \quad i \in S & \\
p(Q)-\nabla p(Q) Q \leq \sum_{i \in S} \eta_{i} q_{i}^{0}, \forall Q \geq 0, \\
u_{i}-\alpha \geq-c_{i}, \quad i \in S \\
\alpha \geq \nabla p(Q), \forall Q \geq 0 \\
u_{i} \geq 0, \alpha \geq 0
\end{array}
$$

where $\lambda_{i}, \eta_{i}, u_{i}$ and $\alpha$ are the variables of the dual problem (30). Denote by $\left(\lambda^{*}, \eta^{*}, u^{*}, \alpha^{*}\right)$ an optimal solution to the dual problem (30) with $S=N$. Define

$$
l_{i}=\lambda_{i}^{*} q_{i}^{0}, i \in N
$$

Proposition 8 The vector $l=\left(l_{1}, l_{2}, \ldots, l_{n}\right)$ defined by Eq. (31) is a profit allocation policy in the core of the capacity pooling game $(N, \pi)$ with a convex quality reward, if $\eta^{*} \geq 0$.

Given a convex quality reward set by an employer, Proposition 8 provides a simple way to compute a fair profit allocation policy for a workers coalition without a refund by solving the dual of a programming problem. Similarly, the condition $\eta^{*} \geq 0$ is also required in Proposition 8, which guarantees the proposed allocation policy $l=\left(l_{1}, l_{2}, \ldots, l_{n}\right)$ in the core of the capacity pooling game $(N, \pi)$ with a convex quality reward. 


\subsection{Concave investment cost and convex quality reward}

For any workers coalition $S \subseteq N$, the concave investment cost and convex quality reward structures are analyzed in Sects. 4.2 and 4.3, respectively. But in reality, these two cases often occur at the same time in crowdsourcing services; that is, the economies of scale and quality incentives appear simultaneously. As such, we focus on the capacity pooling game with a concave investment cost as well as a convex quality reward in this subsection. For each player $i$ of a workers coalition $S \subseteq N$, denote by $c_{i}(\cdot)$ a concave investment cost function of the investment level and $p(\cdot)$ a convex quality reward function of the quality level. We assume that the concave investment cost function $c_{i}(\cdot)$ and the convex quality reward function $p(\cdot)$ are continuously differentiable.

We first consider the existence of a refund, the characteristic profit function $\pi_{r}(S)$, for any workers coalition $S \subseteq N$, with a concave investment cost and a convex quality reward is given by

$$
\begin{aligned}
& \pi_{r}(S)=\max _{Q, q_{i}} p(Q)-\sum_{i \in S} c_{i}\left(q_{i}\right)-\varphi\left(Q-Q_{r}\right)^{+} \\
& \text {s.t. } \quad q_{i} \leq q_{i}^{0}, \quad i \in S, \\
& Q \leq \sum_{i \in S} q_{i}, \\
& Q \geq 0, q_{i} \geq 0 .
\end{aligned}
$$

Introducing a variable $d$ so that problem (32) is converted into a two-stage programming problem

$$
\begin{aligned}
& -\pi_{r}(S)=\min _{q_{i}, Q} \sum_{i \in S} c_{i}\left(q_{i}\right)+r(Q)+l(q, Q) \\
& \text { s.t. } \quad q_{i} \leq q_{i}^{0}, i \in S, \\
& Q-\sum_{i \in S} q_{i} \leq 0, \\
& Q \geq 0, q_{i} \geq 0,
\end{aligned}
$$

where $q=\left(q_{i}\right)_{i \in S}, r(Q)=-p(Q)$ and $l(q, Q)$ is defined as

$$
\begin{aligned}
& l(q, Q)=\min _{d} \varphi d \\
& \text { s.t. } \quad Q-d \leq Q_{r}, d \geq 0 .
\end{aligned}
$$

Next, we pay attention to solving the duality result of the two-stage programming problem (33) and propose a profit allocation policy in the core of the capacity pooling game $\left(N, \pi_{r}\right)$ with the concave investment cost and convex quality reward structures using the duality theory. For this purpose, we define

$$
\Psi^{*}=\min _{x_{i} \in \Delta, y \in \Lambda} \sum_{i \in S} c_{i}\left(x_{i}\right)+r(y)+l(x, y),
$$


where $c_{i}(\cdot)$ and $r(\cdot)$ are both concave functions with $c_{i}(0)=0$ and $r(0)=0$, and $\Delta=\left\{q_{i} \mid q_{i} \leq q_{i}^{0}, i \in S\right\}, \Lambda=\{Q \mid Q \geq 0\}$. Denote by $\nabla c_{i}(\cdot)$ and $\nabla r(\cdot)$ supergradients of the concave functions $c_{i}(\cdot)$ and $r(\cdot)$, respectively. Therefore, by Lemma 3, we have

$$
c_{i}\left(x_{i}\right)=\min _{q_{i} \in \Delta} c_{i}\left(q_{i}\right)+\nabla c_{i}\left(q_{i}\right)\left(x_{i}-q_{i}\right) \text { and } r(y)=\min _{Q \in \Lambda} r(Q)+\nabla r(Q)(y-Q) .
$$

Then, we have

$$
\begin{aligned}
\Psi^{*} & =\min _{x_{i} \in \Delta, y \in \Lambda} \min _{q_{i} \in \Delta, Q \in \Lambda} \sum c_{i \in S}\left(q_{i}\right)+\nabla c_{i}\left(q_{i}\right)\left(x_{i}-q_{i}\right)+r(Q)+\nabla r(Q)(y-Q)+l(x, y) \\
& =\min _{q_{i} \in \Delta, Q \in \Lambda} \sum_{i \in S} c_{i}\left(q_{i}\right)-\nabla c_{i}\left(q_{i}\right) q_{i}+r(Q)-\nabla r(Q) Q+\min _{x_{i} \in \Delta, y \in \Lambda} \sum_{i \in S} \nabla c_{i}\left(q_{i}\right) x_{i}+\nabla r(Q) y+l(x, y) .
\end{aligned}
$$

Consequently, for any $q_{i} \in \Delta$ and $Q \in \Lambda$, we gain that

$$
\begin{aligned}
& \Psi^{*}=\max \Psi \\
& \text { s.t. } \quad \Psi \leq \sum_{i \in S} c_{i}\left(q_{i}\right)-\nabla c_{i}\left(q_{i}\right) q_{i}+r(Q)-\nabla r(Q) Q+\min _{x_{i} \in \Delta, y \in \Lambda} \sum_{i \in S} \nabla c_{i}\left(q_{i}\right) x_{i}+\nabla r(Q) y+l(x, y) .
\end{aligned}
$$

Moreover, for any given $q_{i}$ and $Q$, it is noted that the minimization problem

$$
\min _{x_{i} \in \Delta, y \in \Lambda} \sum_{i \in S} \nabla c_{i}\left(q_{i}\right) x_{i}+\nabla r(Q) y+l(x, y)
$$

is a two-stage linear programming problem, whose duality result is given by

$$
\begin{aligned}
& \max _{t_{i}, \alpha_{1}, \beta_{1}} \sum_{i \in S} t_{i} q_{i}^{0}+\beta_{1} Q_{r} \\
& \text { s.t. } \quad t_{i}-\alpha \leq \nabla c_{i}\left(q_{i}\right), i \in S, \\
& \alpha+\beta_{1} \leq \nabla r(Q),-\beta_{1} \leq \varphi, \\
& t_{i} \leq 0, \alpha \leq 0, \beta_{1} \leq 0,
\end{aligned}
$$

Therefore, we have

$$
\begin{aligned}
& \Psi^{*}=\max \Psi \\
& \text { s.t. } \quad \Psi \leq \sum_{i \in S} c_{i}\left(q_{i}\right)-\nabla c_{i}\left(q_{i}\right) q_{i}+r(Q)-\nabla r(Q) Q+\sum_{i \in S} t_{i} q_{i}^{0}+\beta_{1} Q_{r}, \forall q_{i} \in \Delta, \forall Q \in \Lambda, \\
& t_{i}-\alpha \leq \nabla c_{i}\left(q_{i}\right), i \in S, \forall q_{i} \in \Delta, \\
& \alpha+\beta_{1} \leq \nabla r(Q), \forall Q \in \Lambda,-\beta_{1} \leq \varphi, \\
& t_{i} \leq 0, \alpha \leq 0, \beta_{1} \leq 0 .
\end{aligned}
$$

Moreover, one can define variables $\rho_{i}$ and $\beta_{2}$ for any given $q_{i}$ and $Q$ so that

$$
\sum_{i \in S} c_{i}\left(q_{i}\right)-\nabla c_{i}\left(q_{i}\right) q_{i}+r(Q)-\nabla r(Q) Q \geq \sum_{i \in S} \rho_{i} q_{i}^{0}+\beta_{2} Q_{r} .
$$

We further introduce $\lambda_{i}$ and $\beta$ so that $\lambda_{i} \leq \rho_{i}+t_{i}$ and $\beta \leq \beta_{1}+\beta_{2}$. Hence, the dual of the two-stage programming problem (33) is obtained that 


$$
\begin{aligned}
& \max \sum_{i \in S} \lambda_{i} q_{i}^{0}+\beta Q_{r} \\
& \text { s.t. } \quad \lambda_{i} \leq \rho_{i}+t_{i}, i \in S, \beta \leq \beta_{1}+\beta_{2}, \\
& \sum_{i \in S} \rho_{i} q_{i}^{0}+\beta_{2} Q_{r} \leq \sum_{i \in S} c_{i}\left(q_{i}\right)-\nabla c_{i}\left(q_{i}\right) q_{i}+r(Q)-\nabla r(Q) Q, \forall q_{i} \in \Delta, \forall Q \in \Lambda, \\
& t_{i}-\alpha \leq \nabla c_{i}\left(q_{i}\right), i \in S, \forall q_{i} \in \Delta, \alpha+\beta_{1} \leq \nabla r(Q), \forall Q \in \Lambda,-\beta_{1} \leq \varphi, \\
& t_{i} \leq 0, \alpha \leq 0, \beta_{1} \leq 0,
\end{aligned}
$$

where $\lambda_{i}, \rho_{i}, t_{i}, \alpha, \beta, \beta_{1}$ and $\beta_{2}$ are the variables of dual problem (35). The intuition of dual problem (35) is similar to the dual problem (5). The main difference between the two is the concave capacity investment cost of each worker and the convex quality reward set by the employer.

Denote by $\left(\lambda^{*}, \rho^{*}, t^{*}, \alpha^{*}, \beta^{*}, \beta_{1}^{*}, \beta_{2}^{*}\right)$ an optimal solution to the dual problem (35) with $S=N$. Define for any $i \in N$

$$
l_{r i}=-\left(\lambda_{i}^{*} q_{i}^{0}+\frac{\beta^{*} Q_{r}}{n}\right) .
$$

Proposition 9 The vector $l_{r}=\left(l_{r 1}, l_{r 2}, \ldots, l_{r n}\right)$ given by Eq. (36) is an allocation policy in the core of the capacity pooling game $\left(N, \pi_{r}\right)$ with the concave investment cost and convex quality reward structures, if $\rho^{*} \leq 0$ and $\beta_{2}^{*} \leq 0$.

When both the economies of scale and quality incentives simultaneously emerge, Proposition 9 provides a simple way to compute a profit allocation policy in the core of the capacity pooling game $\left(N, \pi_{r}\right)$ by solving the dual of a two-stage programming problem. In other words, a fair profit allocation policy for the workers coalition with a refund can be defined by any given optimal dual solution. We would like to point out that these conditions $\rho^{*} \leq 0$ and $\beta_{2}^{*} \leq 0$ required in Proposition 9 ensure that the profit allocation policy given by (36) is fair. Moreover, the nonemptiness of the core is proved by Proposition 9 so that the resulting capacity pooling game $\left(N, \pi_{r}\right)$ with the concave investment cost and convex quality reward structures is balanced [4].

Next, we consider the absence of a refund. For any workers coalition $S \subseteq N$ without a refund, the characteristic profit function $\pi(S)$ with a concave investment cost and a convex quality reward is defined as

$$
\begin{aligned}
& \pi(S)=\max _{Q, q_{i}} p(Q)-\sum_{i \in S} c_{i}\left(q_{i}\right) \\
& \text { s.t. } \quad q_{i} \leq q_{i}^{0}, i \in S, \\
& Q \leq \sum_{i \in S} q_{i}, \\
& Q \geq 0, q_{i} \geq 0 .
\end{aligned}
$$

The dual problem of (37) follows directly from the above similar analysis, which is given by 


$$
\begin{aligned}
& \min \sum_{i \in S}-\lambda_{i} q_{i}^{0} \\
& \text { s.t. } \quad \lambda_{i} \leq \rho_{i}+t_{i}, i \in S, \\
& \sum_{i \in S} c_{i}\left(q_{i}\right)-\nabla c_{i}\left(q_{i}\right) q_{i}+r(Q)-\nabla r(Q) Q \geq \sum_{i \in S} \rho_{i} q_{i}^{0}, \forall q_{i} \in \Delta, \forall Q \in \Lambda, \\
& t_{i}-\alpha \leq \nabla c_{i}\left(q_{i}\right), i \in S, \forall q_{i} \in \Delta, \\
& \alpha \leq \nabla r(Q), \forall Q \in \Lambda, \\
& t_{i} \leq 0, \alpha \leq 0
\end{aligned}
$$

where $\lambda_{i}, \rho_{i}, t_{i}$ and $\alpha$ are the dual variables of problem (38). Denote by $\left(\lambda^{*}, \rho^{*}, t^{*}, \alpha^{*}\right)$ an optimal solution to the dual problem (38) with $S=N$. Define

$$
l_{i}=-\lambda_{i}^{*} q_{i}^{0}, i \in N
$$

Proposition 10 The vector $l=\left(l_{1}, l_{2}, \ldots, l_{n}\right)$ defined by Eq. (39) is an allocation policy in the core of the capacity pooling game $(N, \pi)$ with a concave investment cost and a convex quality reward, if $\rho^{*} \leq 0$.

Based on the duality theory, Proposition 10 gives a fair profit allocation policy for players of the workers coalition without a refund facing the economies of scale and quality incentives. We remark that the condition $\rho^{*} \leq 0$ required in Proposition 10 guarantees that the proposed allocation policy is in the core of the capacity pooling game $(N, \pi)$ with a concave investment cost and a convex quality reward.

\section{Extensions and discussions}

So far, the capacity pooling games with the linear and nonlinear structures in the bidding mode have been analyzed in detail. In this section, we mainly discuss two other modes of crowdsourcing service, i.e., the employment mode and the contrast mode.

\subsection{Capacity pooling game in the employment mode}

In the employment mode, every workers coalition first shows their own information on a crowdsourcing platform including the service items, service prices, service experiences, etc. Then, an employer actively seeks out a suitable workers coalition from the corwdsourcing platform to fulfill own task. Once the task is completed by the appointed workers coalition, the employer will pay the negotiated reward to the workers coalition. Similarly, if the realized quality of the answer submitted by the workers coalition is less than the submitted quality, the employer could ask the appointed workers coalition to refund part (or all) of the reward. It is worth noting 
that the types of crowdsourcing tasks in the employment mode are similar to those in the bidding mode.

Obvious to observe that the employment mode is different from the bidding mode at the beginning of the crowdsourcing service. In the employment mode, an employer forwardly looks for a suitable workers coalition to meet the demand of own task on the crowdsourcing platform relying on the information shown by workers coalitions. But different things happen in the bidding mode, an employer first announces own task on the crowdsourcing platform, and then some workers coalitions begin to bid for this task depending on their own abilities after observing the crowdsourcing task. Quite notably, the employment mode becomes similar to the bidding mode once certain workers coalition is employed. Therefore, the characteristic profit function $\pi_{r}(S)(\pi(S))$ for any workers coalition $S \subseteq N$ with a refund (without a refund) in the employment mode is exactly the same as that of the bidding mode. Obviously, the profit allocation policies for players of the workers coalition in the employment mode follow directly from the results given in the bidding mode. For brevity, we will not repeat again.

\subsection{Capacity pooling game in the contrast mode}

In the contrast mode, multiple workers coalitions interested in the task posted on the crowdsourcing platform by an employer first submit their own answers associated with this crowdsourcing task. Then, the employer assess these submitted answers and choose one or more as the selected answer(s). We would like to point out that the contrast mode is more suitable for some creative tasks, for instance, advertising design, marketing service, brand planning and industrial design, which obviously distinguish those tasks served in the bidding and employment modes.

For any winning workers coalition $S \subseteq N$ in the contrast mode, we assume that the capacity investment cost function of every player $i \in S$ is linear, i.e., $c_{i}\left(q_{i}\right)=c_{i} q_{i}$, where $c_{i}$ is unit investment cost; that is, the economies of scale is neglected. The reward for the winning workers coalition $S \subseteq N$ is denoted by $P$. We now focus on the allocation issue of the winning workers coalition in the contrast mode. For any winning workers coalition $S \subseteq N$ with a refund, the characteristic profit function $\pi_{r}(S)$ in the contrast mode is given by

$$
\begin{aligned}
& \pi_{r}(S)=\max _{Q, q_{i}} P-\sum_{i \in S} c_{i} q_{i}-\varphi\left(Q-Q_{r}\right)^{+} \\
& \text {s.t. } \quad q_{i} \geq \underline{q}_{i}, i \in S, \\
& Q \leq \sum_{i \in S} q_{i}, \\
& Q \geq 0 .
\end{aligned}
$$

We introduce a variable $d$ so that problem (40) is equivalent to 


$$
\begin{aligned}
& -\min _{Q, q_{i}} \sum_{i \in S} c_{i} q_{i}+\varphi d-P \\
& \text { s.t. } \quad q_{i} \geq \underline{q}_{i}, i \in S, \\
& \sum_{i \in S} q_{i}-Q \geq 0, \\
& d-Q \geq-Q_{r}, \\
& Q \geq 0, \quad d \geq 0 .
\end{aligned}
$$

It is easy to see that the dual of problem (40) is

$$
\begin{aligned}
& \min _{\lambda_{i}, \alpha, \beta} P-\sum_{i \in S} \lambda_{i} \underline{q}_{i}+\beta Q_{r} \\
& \text { s.t. } \quad \lambda_{i}+\alpha \leq c_{i}, \quad i \in S, \\
& -\alpha-\beta \leq 0, \quad \beta \leq \varphi, \\
& \lambda_{i} \geq 0, \quad \alpha \geq 0, \quad \beta \geq 0,
\end{aligned}
$$

where $\lambda_{i}, \alpha$ and $\beta$ are the decision variables of the dual problem (41). Denote by $\left(\lambda^{*}, \alpha^{*}, \beta^{*}\right)$ an optimal solution to the dual problem (41) with $S=N$. Define for any $i \in N$,

$$
l_{r i}=\frac{P+\beta^{*} Q_{r}}{n}-\lambda_{i}^{*} \underline{q}_{i} .
$$

Proposition 11 The vector $l_{r}=\left(l_{r 1}, l_{r 2}, \ldots, l_{r n}\right)$ defined by Eq. (42) is an allocation policy in the core of the capacity pooling game $\left(N, \pi_{r}\right)$ in the contrast mode.

When there is a refund in the contrast mode, Proposition 11 takes advantage of a constructive proof to show the non-emptiness of the core of the capacity pooling game $\left(N, \pi_{r}\right)$ in the contrast mode. In addition, Proposition 11 provides a fair profit allocation policy for the workers coalition with a refund in the contrast mode using the duality theory. We now turn our attention to another case where there is no refund in the contrast mode. In this case, the characteristic profit function $\pi(S)$ for any winning workers coalition $S \subseteq N$ is defined as

$$
\begin{aligned}
& \pi(S)=\max _{Q, q_{i}} P-\sum_{i \in S} c_{i} q_{i} \\
& \text { s.t. } \quad q_{i} \geq \underline{q}_{i}, \quad i \in S, \\
& Q \leq \sum_{i \in S} q_{i}, \\
& Q \geq 0 .
\end{aligned}
$$

The duality result of problem (43) is acquired immediately, which is given by 


$$
\begin{aligned}
& \min _{\lambda_{i}, \alpha} P+\sum_{i \in S} \lambda_{i} \underline{q}_{i} \\
& \text { s.t. } \quad \lambda_{i}+\alpha \geq-c_{i}, i \in S, \\
& \lambda_{i} \leq 0, \quad \alpha \leq 0,
\end{aligned}
$$

where $\lambda_{i}$ and $\alpha$ are the dual variables of problem (44). Denote by $\left(\lambda^{*}, \alpha^{*}\right)$ an optimal solution to the dual problem (44) with $S=N$. Define

$$
l_{i}=\frac{P}{n}+\lambda_{i}^{*} \underline{q}, i \in N
$$

Proposition 12 The vector $l=\left(l_{1}, l_{2}, \ldots, l_{n}\right)$ given by Eq. (45) is an allocation policy in the core of the capacity pooling game $(N, \pi)$ in the contrast mode.

When there is no refund in the contrast mode, Proposition 12 illustrates that the profit allocation policy defined by (45) is fair based on the duality theory. Next, we analyze the allocation issue for the failed workers coalition in the contrast mode. For any failed workers coalition $S \subseteq N$, it is neither to gain any reward from an employer nor to exist any refund, the characteristic profit function $\pi(S)$ is defined as

$$
\begin{aligned}
& \pi(S)=\max _{Q, q_{i}}-\sum_{i \in S} c_{i} q_{i} \\
& \text { s.t. } \quad q_{i} \geq \underline{q}_{i}, \quad i \in S, \\
& Q \leq \sum_{i \in S} q_{i}, \\
& Q \geq 0 .
\end{aligned}
$$

The dual of problem (46) is easily obtained that

$$
\begin{aligned}
& \min _{\lambda_{i}, \alpha} \quad \sum_{i \in S} \lambda_{i} \underline{q}_{i} \\
& \text { s.t. } \quad \lambda_{i}+\alpha \geq-c_{i}, \quad i \in S, \\
& \lambda_{i} \leq 0, \quad \alpha \leq 0,
\end{aligned}
$$

where $\lambda_{i}$ and $\alpha$ are the decision variables of the dual problem (47). Denote by $\left(\lambda^{*}, \alpha^{*}\right)$ an optimal solution to the dual problem (47) with $S=N$. Define for any $i \in N$

$$
l_{i}=\lambda_{i}^{*} \underline{q}_{i}
$$

Proposition 13 The vector $l=\left(l_{1}, l_{2}, \ldots, l_{n}\right)$ defined by Eq. (48) is a core allocation policy for the failed workers coalition $N$ in the contrast mode. 
As analyzed, the failed workers coalition in the contrast mode also incurs the cost of submitting an answer. For the failed workers coalition $N$, Proposition 13 illustrates that the allocation policy defined by (48) in the core of the capacity pooling game $(N, \pi)$ based on the duality theory. Interestingly, the proposed allocation policy for the failed workers coalition $N$ in the contrast mode can be easily computed by solving the dual of a linear programming problem.

All in all, in this section, we mainly focus on the allocation issues for the workers coalition in the contrast mode under an assumption that the capacity investment cost function is linear. Naturally, a question is raised that how to design a fair allocation policy in the contrast mode when facing the economies of scale. It is appropriate to point out that the analytical scheme of considering the economies of scale is similar to that given in Sect. 4, so we omit it for brevity.

\section{Numerical analysis}

In this section, we employ numerical analyses to examine the proposed main results in this paper and conduct sensitivity analyses of parameters. It should be noted that we mainly focus on the bidding mode in this section, and the employment mode and contrast mode can be analyzed similarly, so we omit them for brevity.

\subsection{The core allocation policy}

Considering a crowdsourcing service, where an employer posts a task in a crowdsourcing platform. Then, three independent workers interested in the posted crowdsourcing task form a workers coalition, denoted by $N=\{1,2,3\}$, to jointly work on this task. Finally, the workers coalition $N$ obtains a reward from the employer.

First, we pay attention to the capacity pooling game with a linear investment cost and quality reward. The parameters throughout this section are given by

$$
q_{1}^{0}=10, q_{2}^{0}=15, q_{3}^{0}=12, Q_{r}=3, p=5, c_{1}=1, c_{2}=1.5, c_{3}=1.2, \varphi=1 .
$$

Immediately, by Proposition 2 and Proposition 4, we gain fair profit allocation policies for the workers coalition $N$ associated with the cases of a refund and no refund, respectively. These results are summarized in Table 1.

Under the assumption of a linear investment cost and quality reward, Table 1 gives fair profit allocation policies for players of the workers coalition when there is a refund and no refund, respectively. It is easy to verify that $l_{r i}<l_{i}$ for worker $i \in N$ from Table 1. In other words, each worker $i \in N$ will be allocated a better profit in the case of no refund, which motivates the workers coalition $N$ to submit a reliable answer associated with their interested crowdsourcing task.

Second, we focus on the capacity pooling game with a concave investment cost. Denote by $c_{i}\left(q_{i}\right)=6 q_{i}-q_{i}^{2}$ the concave investment cost function of each worker $i \in N$. According to Proposition 5 and Proposition 6, we directly obtain fair profit 
Table 1 The allocation policy with a linear investment cost and quality reward

\begin{tabular}{llll}
\hline Allocation policy & Worker 1 & Worker 2 & Worker 3 \\
\hline$l_{r}$ & 31.00 & 38.50 & 34.60 \\
$l$ & 40.00 & 52.50 & 45.6 \\
\hline
\end{tabular}

allocation policies when there is a refund and no refund, respectively, which are given by Table 2 .

Under the economies of scale, it is obvious to see that the allocated profit of each worker $i \in N$ shown in Table 2 is more than that given in Table 1 . Besides, Table 2 also demonstrates that $l_{i}>l_{r i}$ for worker $i \in N$.

Third, we analyze the capacity pooling game with a convex quality reward. Denote by $p(Q)=3 Q^{2}-6 Q$ the convex quality reward associated with the submitted quality. From Proposition 7 and Proposition 8, fair profit allocation policies in different cases are directly obtained.

Compared with Table 1, Table 3 demonstrates that each worker $i \in N$ is allocated a better profit when an employer provides quality incentives, which motivates workers to cooperate and jointly submit a higher quality answer.

Finally, we turn our attention to the capacity pooling game with a concave investment cost and a convex quality reward. For ease of comparison, the concave investment cost function and the convex quality reward function here are adopted the same ones discussed above, that is, $c_{i}\left(q_{i}\right)=6 q_{i}-q_{i}^{2}$ for each worker $i \in N$ and $p(Q)=3 Q^{2}-6 Q$. In this case, based on Proposition 9 and Proposition 10, we immediately gain fair profit allocation polices when there is a refund and no refund, respectively, which is shown in Table 4.

Obviously, when both the economies of scale and quality incentives exist, each worker $i \in N$ is allocated a better profit presented in Table 4 than those given in Tables 1, 2 and 3 whether there is a refund or not.

\subsection{The sensitivity analyses}

As discussed previously, the parameters of unit investment cost, quality reward and refund cost all play particularly important roles in the fair allocation policy proposed in this paper. In this subsection, we mainly focus on the impact of theses parameters

Table 2 The allocation policy with a concave investment cost

\begin{tabular}{llll}
\hline Allocation policy & Worker 1 & Worker 2 & Worker 3 \\
\hline$l_{r}$ & 35.00 & 58.00 & 44.20 \\
$l$ & 44.00 & 72.00 & 55.20 \\
\hline
\end{tabular}

Table 3 The allocation policy with a convex quality reward

\begin{tabular}{llll}
\hline Allocation policy & Worker 1 & Worker 2 & Worker 3 \\
\hline$l_{r}$ & 41.00 & 53.50 & 46.60 \\
$l$ & 50.00 & 67.50 & 57.60 \\
\hline
\end{tabular}


Table 4 The allocation policy with a concave investment cost and a convex quality reward

\begin{tabular}{llll}
\hline Allocation policy & Worker 1 & Worker 2 & Worker 3 \\
\hline$l_{r}$ & 43.00 & 69.00 & 52.80 \\
$l$ & 56.00 & 90.00 & 69.60 \\
\hline
\end{tabular}

on the proposed fair allocation policy under linear structures. Fortunately, the other cases can be analyzed in a similar way.

As mentioned, the parameters $c, p$ and $\varphi$ denote unit investment cost, unit quality reward and unit refund cost, respectively. We now analyze the impact of unit investment $\operatorname{cost} c$ on the fair allocation policies for three players in the workers coalition.

Figure 1 depicts the allocated profit for each player of the workers coalition with respect to different unit investment cost. It is easy to verify that the allocated profit $l_{r i}\left(l_{i}\right)$ for each worker $i$ is negatively correlated with unit investment cost $c$. That is to say, the larger the unit investment cost, the smaller the allocated profit for each worker, which is in line with the proposed results in this paper. The reason for this phenomenon is that when the reward is kept unchanged, an increase in the investment cost leads to a decrease in the profit of the workers coalition. Then, we investigate the impact of unit quality reward on the profit allocation policies for three workers.

Obviously, Fig. 2 illustrates that the allocated profit of each worker increases as the unit quality reward changes. In other words, the higher the unit quality reward $p$, the larger the allocated profit $l_{r i}\left(l_{i}\right)$ for each worker $i$, which is consistent with our results. This is because an increase in the unit quality reward would bring about an increase in the profit of the workers coalition. Thus, increasing the quality reward would benefit all players of the workers coalition. Moreover, we investigate the impact of unit refund cost on the profit allocation policies for three workers.
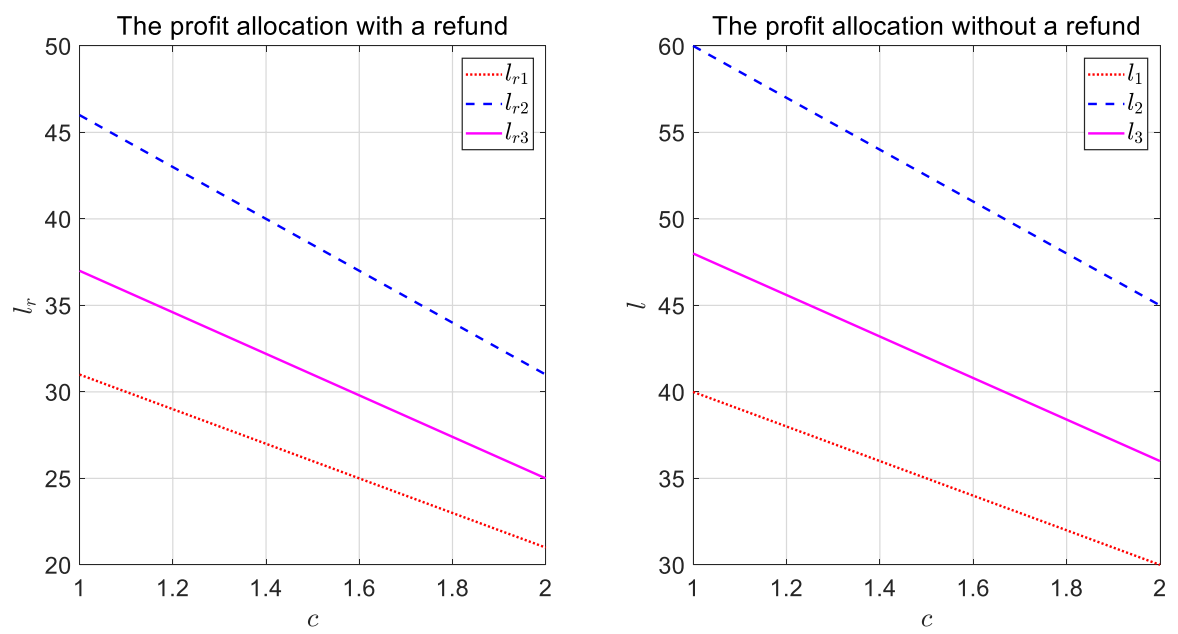

Fig. 1 The profit allocation as unit investment cost $c$ changes 

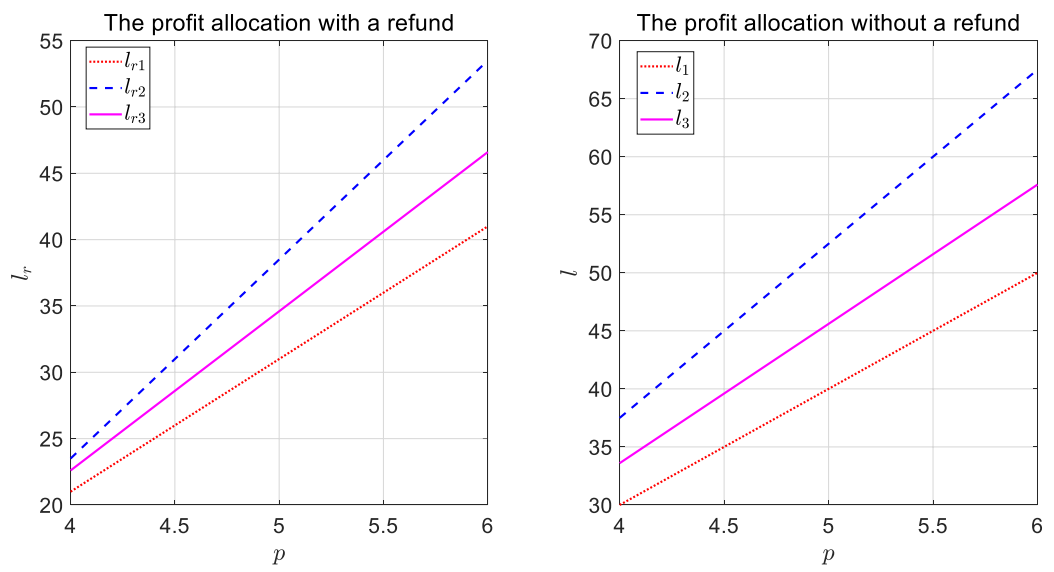

Fig. 2 The profit allocation as unit quality reward $p$ changes

From Fig. 3, we observe that when there is a refund, the allocated profit $l_{r i}$ of each worker $i \in N$ decreases when the unit refund $\varphi$ increases. Namely, the allocated profit and the unit refund are negatively correlated, which illustrates the results proposed by this paper. The reason for this phenomenon is that the unit refund has a negative effect on the profit of the workers coalition. In addition, the changes in the three different profit curves are clearly similar, which means that the unit refund $\varphi$ has similar impact on the allocated profit of each worker $i \in N$. Furthermore, the impact of the nameplate capacity on the profit allocation policy is given as follows.

Figure 4 illustrates that the allocated profit $l_{r i}\left(l_{i}\right)$ of each worker $i \in N$ increases as the nameplate capacity $q^{0}$ changes. This result means that the greater the

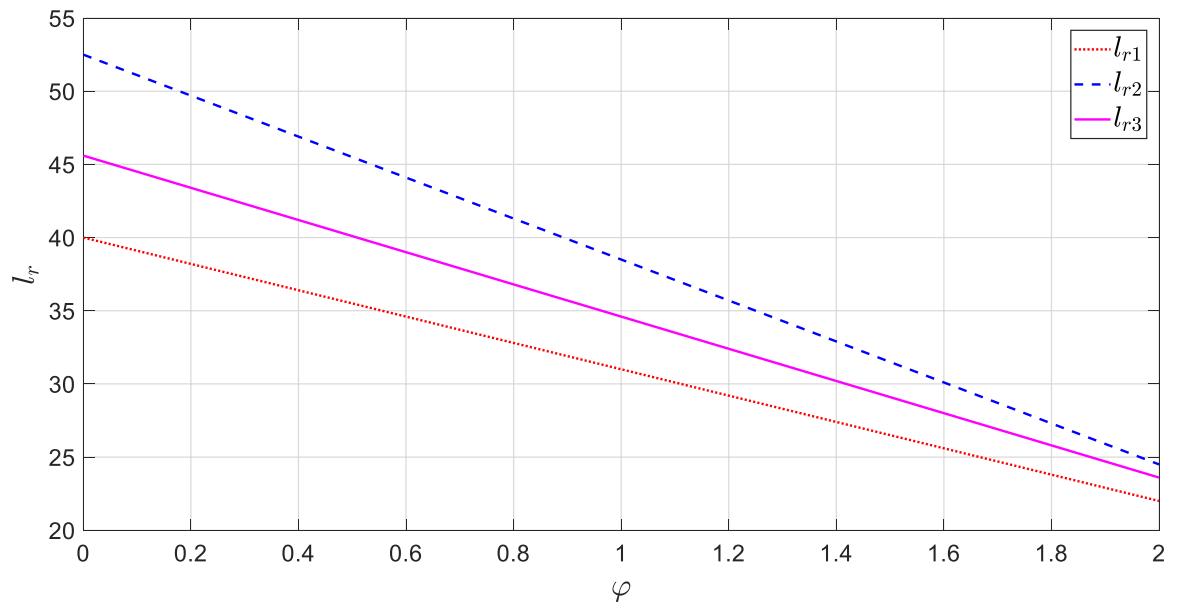

Fig. 3 The profit allocation as unit refund $\varphi$ changes 
nameplate capacity of a worker, the more the allocated profit, the reason for which is that the worker having a greater nameplate capacity makes a bigger contribution to the submitted answer. Obviously, this finding is consistent with the result presented by this paper. Finally, we focus on the impact of the realized quality of a submitted answer on the profit allocation policy for a workers coalition.

By Fig. 5, it is easy to see that the allocated profit $l_{r i}$ of each worker $i \in N$ and the realized quality $Q_{r}$ of a submitted answer are positively correlated; that is, a higher realized quality would bring workers a better profit. This is because a higher realized quality of the submitted answer would lead to a less refund for the workers coalition.

\section{Conclusion}

Crowdsourcing is an online, distributed problem solving and innovative sourcing model, in which members of online communities contribute to carrying out specific tasks. Crowdsourcing accelerates the idea discovery process by expanding the scale and diversity of collective wisdom devoted to problem solving. The emergence of crowdsourcing platforms greatly reduces the costs and broadens the reach of collecting a huge amount of data and information from people all over the world. Thus, crowdsourcing has the potential to accelerate research due to the amount of work that can be concentrated on a single challenge in a short period, which makes it can be a promising tool in health, and in global health in particular. In recent years, there is much evidence to support the effectiveness and the successful use of crowdsourcing applied to global health. Over the last months, the coronavirus disease COVID-19 has spread with unprecedented speed and scale, and the outbreak situation is constantly evolving. The COVID-19 has been one of the largest medical disasters since the Spanish flu pandemic of 1918. During the past few months, different stakeholders (e.g., health care, industry, governments, academics, ordinary people)
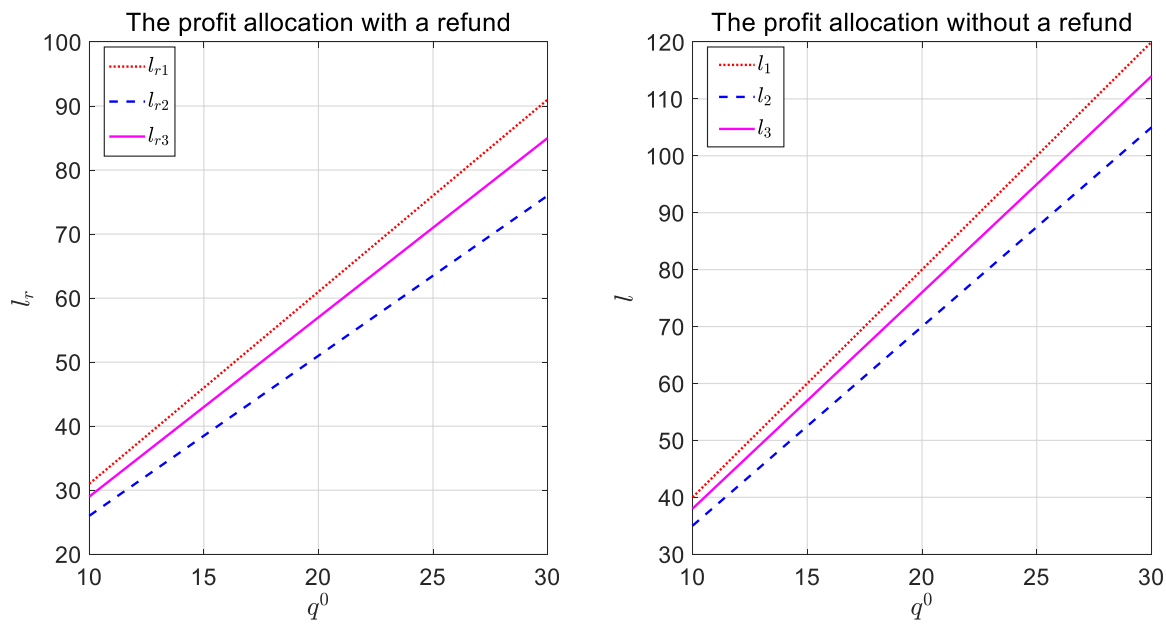

Fig. 4 The profit allocation as the nameplate capacity $q^{0}$ changes 


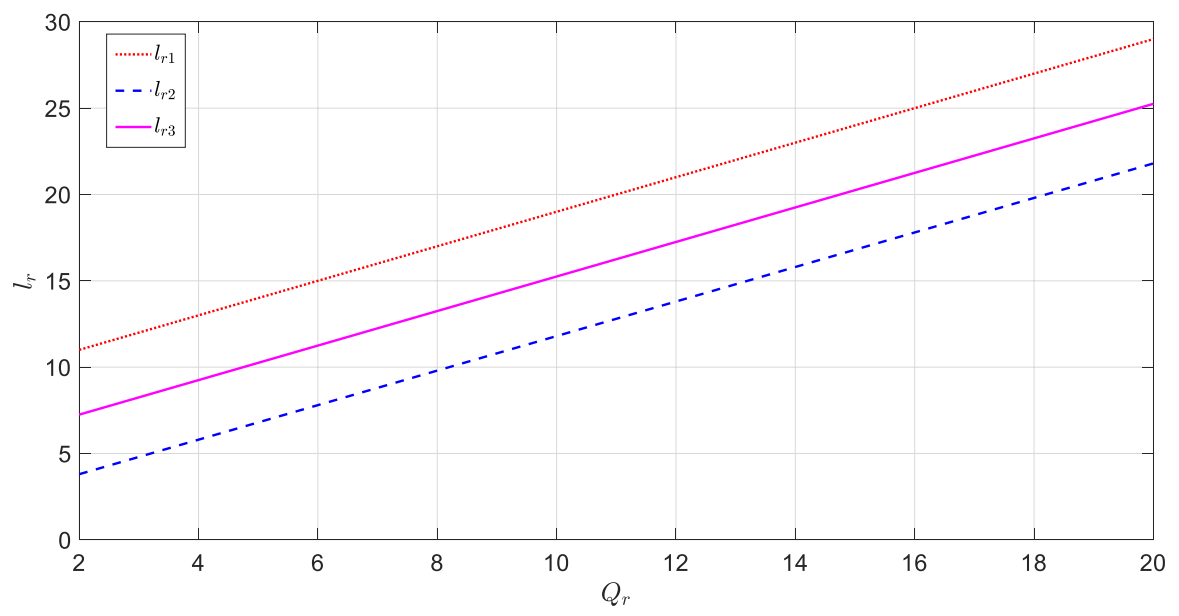

Fig. 5 The profit allocation as the realized quality $Q_{r}$ changes

develop innovative solutions and collaborative infrastructures in the fight against the COVID-19 crisis. Thus, by providing original, actionable, quick, and low-cost solutions to the challenges of the current health and economic crisis, crowdsourcing, harnessing the power of crowds and adopting an open and collaborative approach, can help tackle the COVID-19 pandemic. Interestingly, this paper provides a unified framework of cooperative game for independent workers in crowdsourcing task.

In this paper, we focus on the cooperative behaviors among workers facing a complex or difficult task, and the design of a fair profit allocation policy for a workers coalition in crowdsourcing services. Our paper provides the following main contributions. First, we show that why independent workers should cooperate, and how to design a fair profit allocation policy for a workers coalition in crowdsourcing services. On the one hand, in the bidding mode, whether there is a refund or not, we find that the core of the capacity pooling game with a linear (or concave) investment cost and a linear (or convex) quality reward is nonempty. On the other hand, in both the employment mode and contrast mode, we also find that the corresponding capacity pooling game has a nonempty core. Second, we generate some implications to help workers obtain better profits. For example, workers should try their best to improve their nameplate capacity levels, and reduce their investment costs and refund costs. In the meantime, workers should ensure that the realized quality of a submitted answer is consistent with the submitted quality. Third, we provide a simple and stylized model for workers' cooperation in crowdsourcing services. Thus, our model not only can serve as a reference point for future empirical fieldwork but also can be a useful building block for more complicated models. Finally, we give a unified and parsimonious framework of profit allocation policy for studying the workers' cooperative strategies in crowdsourcing services. The proposed allocation policy is proven to be fair, and so it plays an important role in the sustainable development of workers' cooperative behaviors. 
The findings of this paper illustrate that independent workers can willingly form a coalition to jointly accomplish a complex or difficult crowdsourcing task, and then is allocated a fair profit, which motivates independent workers to cooperate in crowdsourcing services. Moreover, our findings give an incentive for employers to post complex or difficult tasks on the crowdsourcing platform, which can help employers get answers to the crowdsourcing tasks at a lower cost. Additionally, it is easy to verify that, when a workers coalition accomplishes a complex or difficult crowdsourcing task posted by an employer, the crowdsourcing platform can obtain a profit from the employer.

There are several interesting directions for future research in relation to this paper. First, the unit quality reward of the submitted answer is assumed exogenous in this paper, Thus, it would be interesting to extend our proposed duality approach to analyze another case in which the unit quality reward is considered to be endogenous. Second, this paper assume that a workers coalition only undertakes a single crowdsourcing task. Hence, it would also be interesting to conduct research into the design of a fair profit allocation policy when a workers coalition undertakes multiple tasks.

\section{Appendix}

The proof of Lemma 1 It is straightforward to verify that $-\pi(S)$ defined by (4) is equal to the optimal value of (5) based on the strong duality theory of Rockafellar and Wets [30].

The proof of Proposition 1 Consider the case in which the workers coalition has to return partial (or full) reward to the employer, so we have $Q^{*}>Q_{r}$ and $d^{*}>0$. Since $d^{*}$ is the dual variable of the third constraint in (5), the corresponding constraint must hold as equality, i.e., $\beta^{*}=-\varphi$.

It follows that $q_{i}^{*}>0$. Otherwise, the workers coalition cannot submit any answer to the employer. Because the optimal capacity investment level $q_{i}^{*}$ is the dual variable of the first constraint in (5), we have $\lambda_{i}^{*}-\alpha^{*}=c_{i}$ based on the complementary slackness conditions. Observe that $Q^{*}>0$, otherwise, the employer cannot pay any reward to the workers coalition. Since the optimal reward $Q^{*}$ is the dual variable of the second constraint in (5), it follows that $\alpha^{*}+\beta^{*}=-p$. Therefore, we have $\lambda_{i}^{*}=\varphi-p+c_{i}, \alpha^{*}=\varphi-p$ and $\beta^{*}=-\varphi$.

Apply the duality theory of Lemma 1 to the primal problem (4) and dual problem (5), we know that

$$
-p Q^{*}+\sum_{i \in S} c_{i} q_{i}^{*}+\varphi d^{*}=\sum_{i \in S} \lambda_{i}^{*} q_{i}^{0}+\beta^{*} Q_{r} \cdot a
$$

Thus, we obtain directly that $q_{i}^{*}=q_{i}^{0}, Q^{*}=\sum_{i \in S} q_{i}^{0}$ and $d^{*}=\sum_{i \in S} q_{i}^{0}-Q_{r}$.

The proof of Proposition 2 First, by the definition of (6), we derive the following result immediately: 


$$
\sum_{i \in N} l_{r i}=\sum_{i \in N}-\left(\lambda_{i}^{*} q_{i}^{0}+\frac{\beta^{*} Q_{r}}{n}\right)=-\sum_{i \in N}\left(\lambda_{i}^{*} q_{i}^{0}+\frac{\beta^{*} Q_{r}}{n}\right) .
$$

Recall that $\left(\lambda^{*}, \alpha^{*}, \beta^{*}\right)$ is the optimal solution to the dual problem (5) with $S=N$. Then, based on the strong duality theory from Lemma 1 , we have

$$
-\pi_{r}(N)=\sum_{i \in N}\left(\lambda_{i}^{*} q_{i}^{0}+\frac{\beta^{*} Q_{r}}{n}\right) .
$$

As a result,

$$
\sum_{i \in N} l_{r i}=-\sum_{i \in N}\left(\lambda_{i}^{*} q_{i}^{0}+\frac{\beta^{*} Q_{r}}{n}\right)=\pi_{r}(N) .
$$

We claim that the vector $l_{r}=\left(l_{r 1}, l_{r 2}, \ldots, l_{r n}\right)$ defined by (6) is an allocation of the capacity poling game $\left(N, \pi_{r}\right)$.

Then, it is noted that $\left(\lambda^{*}, \alpha^{*}, \beta^{*}\right)$ is a feasible solution to the dual problem (5) with $S \subseteq N$ in the meantime. Therefore, for any collection $S \subseteq N$, substituting the feasible solution $\left(\lambda^{*}, \alpha^{*}, \beta^{*}\right)$ into (5) and combining the vector $l_{r}$ defined by (6), we have

$$
\sum_{i \in S}\left(\lambda_{i}^{*} q_{i}^{0}+\frac{\beta^{*} Q_{r}}{s}\right)=\sum_{i \in S}-l_{r i}=-\sum_{i \in S} l_{r i}
$$

Moreover, from Lemma $1,-\pi_{r}(S)$ is equal to the maximum value of (5), so we derive the following result directly:

$$
\sum_{i \in S}\left(\lambda_{i}^{*} q_{i}^{0}+\frac{\beta^{*} Q_{r}}{s}\right) \leq-\pi_{r}(S), \forall S \subseteq N
$$

That is to say,

$$
\sum_{i \in S} l_{r i} \geq \pi_{r}(S), \quad \forall S \subseteq N
$$

Therefore, combining (49) and (50) together, we show that the profit allocation policy $l_{r}=\left(l_{r 1}, l_{r 2}, \ldots, l_{r n}\right)$ defined by (6)

in the core of the capacity pooling game $\left(N, \pi_{r}\right)$.

The proof of Corollary 1 Proposition 2 shows that the core of the capacity pooling game $\left(N, \pi_{r}\right)$ is nonempty. Thus, from the results of Bondareva [4], we know immediately that the resulting capacity pooling game $\left(N, \pi_{r}\right)$ is balanced.

The proof of Lemma 2 It follows directly from that of Lemma 1.

The proof of Proposition 3 Recall that $Q^{*}$ and $q^{*}$ are the optimal solutions to the primal problem (7), and $\lambda^{*}$ and $\alpha^{*}$ are the optimal dual solutions to the dual problem (8). In this case where there is no refund, it is easy to know that $Q^{*}=Q_{r}$. Observe 
that $q_{i}^{*}>0$, otherwise, the workers coalition $S$ is impossible to provide an answer to the employer. Since the optimal capacity investment level $q_{i}^{*}$ is the dual variable of the first constraint in (8), we have $\lambda_{i}^{*}-\alpha^{*}=-c_{i}$ using the corresponding complementary slackness conditions.

Moreover, it follows that $Q^{*}>0$. Otherwise the employer will not pay any reward to the workers coalition $S$. Because the optimal submitted quality $Q^{*}$ is the dual variable of the second constraint in (8), we get $\alpha^{*}=p$. Next, applying the result of Lemma 2 to the primal problem (7) and dual problem (8), we have

$$
p Q^{*}-\sum_{i \in S} c_{i} q_{i}^{*}=\sum_{i \in S} \lambda_{i}^{*} q_{i}^{0}
$$

Consequently, we derive that $q_{i}^{*}=q_{i}^{0}$ and $Q^{*}=Q_{r}=\sum_{i \in S} q_{i}^{0}$.

The proof of Proposition 4 The proof is similar to that of Proposition 2 and so it is omitted.

The proof of Lemma 3 Denote $y \in D$, by the definition of concavity, we have

$$
F(x+t(y-x)) \geq F(x)+t(F(y)-F(x)) .
$$

Rearranging and dividing by $t>0$, the above inequality reduces to

$$
\frac{F(x+t(y-x))-F(x)}{t} \geq F(y)-F(x) \text {. }
$$

Particularly, let $t \rightarrow 0$, the left side converges to $F^{\prime}(x)(y-x)$. Therefore, for every $y$ in $D$, we have $F(x)+F^{\prime}(x)(y-x) \geq F(y)$.

The proof of Proposition 5 The proof is similar to that of Proposition 2 and so it is omitted.

The proof of Proposition 6 The proof is similar to that of Proposition 2 and so it is omitted.

The proof of Lemma 4 Denote $y$ in $C$. By the definition of convexity, for $t \in(0,1)$, we know that

$$
t G(y)+(1-t) G(x) \geq G(t y+(1-t) x) .
$$

Rearranging the above inequality, we have

$$
t(G(y)-G(x)) \geq G(x+t(y-x))-G(x) .
$$

Dividing by $t>0$, the above inequality is converted as

$$
G(y)-G(x) \geq \frac{G(x+t(y-x))-G(x)}{t} .
$$


Then, let $t \rightarrow 0$, the right side converges to $G^{\prime}(x)(y-x)$. Hence, for every $y \in C$, we gain $G(y) \geq G(x)+G^{\prime}(x)(y-x)$.

The proof of Proposition 7 The proof is similar to that of Proposition 2 and so it is omitted.

The proof of Proposition 8 The proof is similar to that of Proposition 2 and so it is omitted.

The proof of Proposition 9 The proof is similar to that of Proposition 2 and so it is omitted.

The proof of Proposition 10 The proof is similar to that of Proposition 2 and so it is omitted.

The proof of Proposition 11 The proof is similar to that of Proposition 2 and so it is omitted.

The proof of Proposition 12 The proof is similar to that of Proposition 2 and so it is omitted.

The proof of Proposition 13 The proof is similar to that of Proposition 2 and so it is omitted.

Acknowledgements This research was supported by: (i) the National Natural Science Foundation of China under Grants 72071074 and 71671061; and (ii) the Outstanding Young Foundation of Hunan Province Natural Science under Grants 2018JJ1003.

\section{References}

1. Allon, G., \& Babich, V. (2020). Crowdsourcing and crowdfunding in the manufacturing and services sectors. Manufacturing \& Service Operations Management, 22(1), 102-112.

2. Anily, S. (2018). Full characterization of the nonnegative core of some cooperative games. Naval Research Logistics, 65(4), 303-316.

3. Archak, N., \& Sundararajan, A. (2009). Optimal design of crowdsourcing contests. In ICIS 2009 proceedings (p. 200).

4. Bondareva, O. N. (1963). Some applications of the methods of linear programming to the theory of cooperative games. Problemy Kibernetiki, 10, 119-139.

5. Chan, K. W., Li, S. Y., Ni, J., \& Zhu, J. J. (2021). What feedback matters? The role of experience in motivating crowdsourcing innovation. Production and Operations Management, 30(1), 103-126.

6. Chen, P. Y., Pavlou, P., Wu, S., \& Yang, Y. (2021). Attracting high-quality contestants to contest in the context of crowdsourcing contest platform. Production and Operations Management (Forthcoming).

7. Chen, Y., Teck-Hua, H. O., \& Kim, Y. M. (2010). Knowledge market design: A field experiment at google answers. Journal of Public Economic Theory, 12(4), 641-664. 
8. Chen, X., \& Zhang, J. W. (2009). A stochastic programming duality approach to inventory centralization games. Operations Research, 57(4), 840-851.

9. DiPalantino, D., \& Vojnovic, M. (2009). Crowdsourcing and all-pay auctions. In Proceedings of the 10th ACM conference on electronic commerce (pp. 119-128).

10. Ghezzi, A., Gabelloni, D., Martini, A., \& Natalicchio, A. (2018). Crowdsourcing: A review and suggestions for future research. International Journal of Management Reviews, 20(2), 343-363.

11. Ghosh, A., \& McAfee, P. (2012). Crowdsourcing with endogenous entry. In World wide web conference, Lyon, France.

12. Howe, J. (2006). The rise of crowdsourcing. Wired, 14, 1-4.

13. Hu, M., \& Wang, L. (2020). Joint versus separate crowdsourcing contests. Management Science (Forthcoming).

14. Huang, Y., Singh, P. V., \& Mukhopadhyay, T. (2012). Crowdsourcing contests: A dynamic structural model of the impact of incentive structure on solution quality. In Thirty third international conference on information systems.

15. Huang, Y., Vir Singh, P., \& Srinivasan, K. (2014). Crowdsourcing new product ideas under consumer learning. Management Science, 60(9), 2138-2159.

16. Jeon, G. Y. J., Kim, Y. M., \& Chen, Y. (2010). Re-examining price as a predictor of answer quality in an online Q\&A site. In Proceedings of the SIGCHI conference on human factors in computing systems (pp. 325-328). ACM.

17. Jiang, Z., Huang, Y., \& Beil, D. R. (2020). The role of problem specification in crowdsourcing contests for design problems: A theoretical and empirical analysis. Manufacturing \& Service Operations Management (Forthcoming).

18. Kamath, K. Y., \& Caverlee, J. (2011). Transient crowd discovery on the real-time social web. In Proceedings of the fourth ACM international conference on Web search and data mining (pp. 585-594).

19. Karger, D. R., Oh, S., \& Shah, D. (2014). Budget-optimal task allocation for reliable crowdsourcing systems. Operations Research, 62(1), 1-24.

20. Karsten, F., Slikker, M., \& van Houtum, G. J. (2012). Inventory pooling games for expensive, lowdemand spare parts. Naval Research Logistics, 59(5), 311-324.

21. Liu, T. X., Yang, J., Adamic, L. A., \& Chen, Y. (2014). Crowdsourcing with all-pay auctions: A field experiment on task. Management Science, 60(8), 2020-2037.

22. Majchrzak, A., \& Malhotra, A. (2020). Unleashing the crowd collaborative solutions to wicked business and societal problems (pp. 1-302). Palgrave Macmillan.

23. Mo, J., Zheng, Z., \& Geng, X. (2011). Winning crowdsourcing contests: A micro-structural analysis of multi-relational social network. In The 5th workshop on information management (CSWIM), Harbin, China.

24. Nevo, D., \& Kotlarsky, J. (2020). Crowdsourcing as a strategic is sourcing phenomenon: Critical review and insights for future research. The Journal of Strategic Information Systems, 29(4), 101593.

25. Owen, G. (1975). On the core of linear production games. Mathematical Programming, 9(1), $358-370$.

26. Owen, G. (1995). A course in game theory (3rd ed.). Academic Press.

27. Pandey, S. R., Tran, N. H., Bennis, M., Tun, Y. K., Manzoor, A., \& Hong, C. S. (2020). A crowdsourcing framework for on-device federated learning. IEEE Transactions on Wireless Communications, 19(5), 3241-3256.

28. Papanastasiou, Y., Bimpikis, K., \& Savva, N. (2018). Crowdsourcing exploration. Management Science, 64(4), 1727-1746.

29. Peleg, B., \& Sudhölter, P. (2003). Introduction to the theory of cooperative games. Kluwer Academic Publishers.

30. Rockafellar, R., \& Wets, R. (1976). Stochastic convex programming: Relatively complete recourse and induced feasibility. SIAM Journal on Control and Optimization, 14(3), 574-589.

31. Samet, D., \& Zemel, E. (1984). On the core and dual set of linear programming games. Mathematics of Operations Research, 9(2), 309-316.

32. Schlicher, L., Slikker, M., \& Houtum, G. J. V. (2017). Probabilistic resource pooling games. Naval Research Logistics, 64(7), 531-546.

33. Schaarsberg, M. G., Borm, P., Hamers, H., \& Reijnierse, H. (2013). Game theoretic analysis of maximum cooperative purchasing situations. Naval Research Logistics, 60(8), 607-624.

34. Shapley, L. S. (1971). Cores of convex games. International Journal of Game Theory, 1(1), 11-26. 
35. Shi, Z., \& Cao, E. (2020). Contract farming problems and games under yield uncertainty. Australian Journal of Agricultural and Resource Economics, 64(4), 1210-1238.

36. Shubik, M. (1962). Incentives, decentralized control, the assignment of joint costs and internal pricing. Management Science, 8(3), 325-343.

37. Ta, H., Esper, T. L., \& Tokar, T. (2021). Appealing to the crowd: Motivation message framing and crowdsourcing performance in retail operations. Production and Operations Management (Forthcoming).

38. Tamir, A. (1991). On the core of network synthesis games. Mathematical Programming, 50(1), $123-135$.

39. Terwiesch, C., \& Xu, Y. (2008). Innovation contests, open innovation, and multiagent problem solving. Management Science, 54(9), 1529-1543.

40. Tong, Y., Zhou, Z., Zeng, Y., Chen, L., \& Shahabi, C. (2020). Spatial crowdsourcing: A survey. The VLDB Journal, 29(1), 217-250.

41. Wen, Z., \& Lin, L. (2016). Optimal fee structures of crowdsourcing platforms. Decision Sciences, 47(5), 820-850.

42. Wikipedia. (2019). Crowdsourcing. Accessed April 3, 2019. http://en.wikipedia.org/wiki/Crowd sourcing.

43. Yang, Y., Chen, P., \& Paul, P. (2009). Open innovation: An empirical study of online contests. In Proceedings of the 30th international conference on information systems, Phoenix, AZ.

44. Zhao, Y., \& Zhu, Q. (2014). Evaluation on crowdsourcing research: Current status and future direction. Information Systems Frontiers, 16(3), 417-434. 